\title{
A NEW APPROACH TO ATTITUDE STABILITY AND CONTROL FOR LOW AIRSPEED VEHICLES
}

\author{
K.B. Lim, J-Y. Shin, D.D. Moerder $\stackrel{\ddagger}{\dagger}$ E.G. Cooper ${ }^{\S}$ \\ NASA Langley Research Center \\ Hampton, Virginia, USA 23681
}

\begin{abstract}
This paper describes an approach for controlling the attitude of statically unsable thrust-levitated vehicles in hover or slow translation. The large thrust vector that characterizes such vehicles can be modulated to provide control forces and moments to the airframe, but such modulation is accompanied by significant unsteady flow effects. These effects are difficult to model, and can compromise the practical value of thrust vectoring in closedloop attitude stability, even if the thrust vectoring machinery has sufficient bandwidth for stabilization. The stabilization approach described in this paper is based on using internal angular momentum transfer devices for stability, augmented by thrust vectoring for trim and other "outer loop" control functions. The three main components of this approach are: (1) a $z$-body axis angular momentum bias enhances static attitude stability, reducing the amount of control activity needed for stabilization, (2) optionally, gimbaled reaction wheels provide highbandwidth control torques for additional stabilization, or agility, and (3) the resulting strongly coupled system dynamics are controlled by a multivariable controller. A flight test vehicle is described, and nonlinear simulation results are provided that demonstrate the efficacy of the approach.
\end{abstract}

\section{Introduction}

\subsection{Background}

There has been recent interest in flight vehicles, capable of VTOL and hover, that are statically unstable or neutrally stable [1]. Examples of such vehicles include small observation platforms [2], [3] in which an optical sensor is mounted above a levitation-producing ducted fan, and rescue vehicles [4] consisting of a payload-bearing plat-

\footnotetext{
*Senior Research Engineer, Guidance and Controls Branch, kyong.b.lim@nasa.gov

†Staff Scientist, National Institute of Aerospace, $j \cdot y \cdot$ shin@larc.nasa.gov

${ }^{\ddagger}$ Senior Research Engineer, Guidance and Controls Branch, d.d.moerder@larc.nasa.gov

§Senior Research Engineer, Systems Integration Branch, e.g.cooper@larc.nasa.gov
}

form with a number of ducted fans distributed symmetrically about its perimeter in the plane of the platform. In the state of the art, this type of vehicle is controlled by thrust vector variation, possibly with additional aerodynamic features implemented to enhance stability in the presence of gusts [2], [3].

Using thrust vectoring or differential thrusting to stabilize a statically unstable airframe poses two related difficulties. First, because of the airframe's lack of stability, the controller must operate with high authority and bandwidth in order to contain and suppress excursions in attitude. Accurate system models are generally required to successfully design such controllers. The second difficulty is that such models are not available. This is because the active thrust vector variations required for stabilization in the presence of disturbances induces unsteady flow phenomena that distort the control command in a complicated manner that currently defies comprehensive modelling (see for example [5]. These difficulties come together when the control engineer attempts to design the required high-performance controller while ignoring or under-modelling the effect of the unsteady flow phenomena on the closed-loop dynamics These difficulties become more evident for smaller inertia VTOL vehicles because they respond more rapidly to disturbances so that correspondingly more rapid feedback control is necessary for vehicle stabilization.

\subsection{A New Approach}

This paper describes the configuration, dynamics, and control of a statically unstable flight vehicle that levitates by means of thrusters with thrust vectoring devices, but augments the thrust vector control with internal angular momentum transfers to enhance attitude stability and provide secure command tracking performance in the presence of external disturbances, such as wind gusts and in-flight payload variations.

The salient features of such a vehicle are:

- The vertical component of the thrust vector levitates the vehicle.

- The thrust vector is produced by a plurality of thrust generators, such as ducted fans. The direction of the thrust vector is modified by changing the orientation 
of the thrust generators, such as by gimballing, or by diverting their flow. The contributions from the plurality of thrusters produce net forces and moments about the airframe's center of mass that can be used to trim the vehicle and for control. For the example system explored further in the sequel, thrust is provided by four ducted fans, each with axis of rotation fixed parallel to the $z$ body axis, and the thrust direction is modulated by a pair of thrust vectoring vanes under each fan.

- Static attitude stability is enhanced by an angular momentum bias in the $z$ body axis. This bias is produced by a plurality of rotating masses, such as dedicated momentum wheels. Alternatively, when employing fans or impellers whose rotation is aligned with the $z$ body axis for levitation thrust, the $z$ body angular momentum bias can be generated by augmenting their moments of inertia about their rotation axes. This is done by redistributing mass toward, or introducing additional mass near the fan or impeller radius for one or more of the fans or impellers. An example of this is attaching a ring to the tips of a levitation fan.

- Additional attitude control authority is provided by a plurality of control moment gyros (CMGs). These interact with the thrust vector control in jointly generating moments for attitude stabilization; the thrust vectoring system is also used to desaturate the CMGs.

- The control effectors described above are jointly commanded by a control system for stabilization and command tracking, and for desaturation of the CMGs.

The advantages of the above vehicle are:

- The vehicle's open-loop attitude stability is enhanced by the $z$-body axis angular momentum bias. This reduces the authority required from the active stabilization control system, and permits a reduction in the bandwidth of the thrust vectoring control commands.

- It is straightforward to develop accurate control design models of the devices for internal angular momentum exchanges. Because of this, they can be used in high-bandwidth, high-authority control systems. This permits enhanced disturbance rejections and maneuverability.

- The use of internal angular momentum exchange devices relaxes the performance requirements on the thrust vector control effectors, since their role can be reduced to as little as that of providing trim, command tracking, and CMG desaturation.

Figure 1 illustrates the basic differences between the state-of-the-art approach and our new approach.

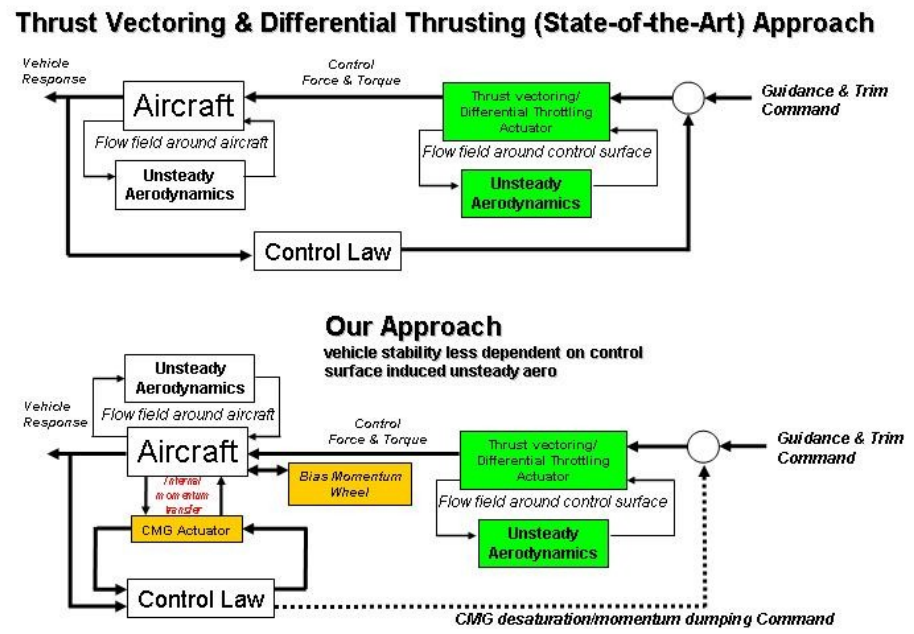

Figure 1: Inner-loop attitude control by differential and thrust vectoring, state-of-the-art (upper), and by internal torques aided by bias momentum, our approach (lower).

\subsection{Outline of Paper}

This paper outlines recent results in our continuing $R \& D$ efforts as first reported in [6]. In the next section, a research vehicle under development at NASA Langley Research Center for maturing this control technology is described, and its equations of motion are given. Section 3 discuss its flight dynamics and control aspects including vehicle trim, gyric stability, and control structure. The sizing of the bias momentum for this new class of "hovering spacecraft" vehicles is also discussed. In section 4, we describe a current flying test platform system under development and outline its simulation-based performance assessment and comparison with a similar vehicle not using momentum exchange devices. Section 5 provides conclusions.

\section{Equations of Motion}

To derive the equations of motion for a generic NFTP system $^{1}$, a Newton-Euler formulation is used, and in particular, the sequence of formulation and notation found in [7], and extended to multiple gimbaled spinning bodies with vane rotational motion for vector thrusting. Only a summary of the results are given here and the interested reader is referred to [8] for details.

Figure 2 shows a schematic of the NFTP describing its main components.

\footnotetext{
1 "Generic" in the sense of a flying platform propelled by multiple ducted-fan and vane system and augmented with multiple wheel system
} 


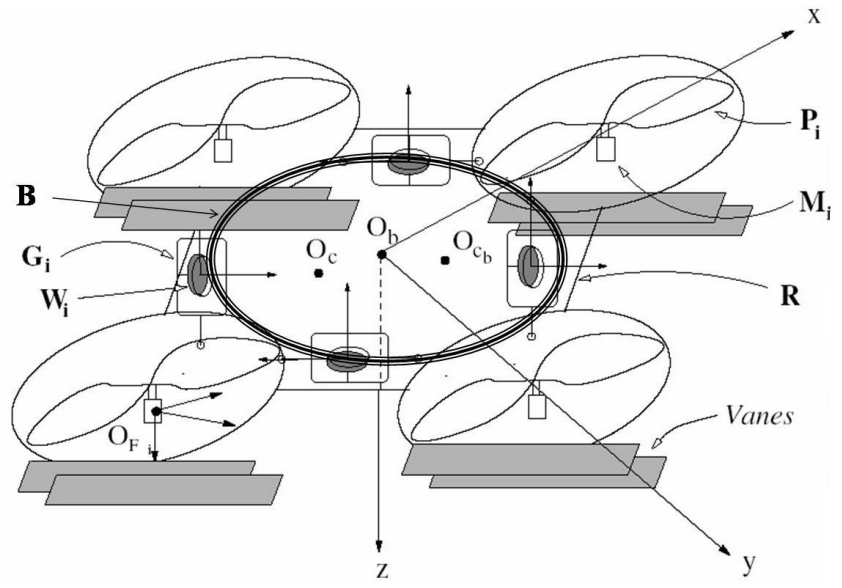

Figure 2: Schematic of NFTP.

$R \quad$ Rigid platform

$F_{i} \quad i$-th Fan: $\operatorname{Propeller}\left(P_{i}\right)+\operatorname{Motor}\left(M_{i}\right)$

$C_{i} \quad i$-th CMG: Wheel $\left(W_{i}\right)+\operatorname{Gimbal}\left(G_{i}\right)$

$B \quad$ Momentum wheel, centered at $O_{b}$

$O_{b} \quad$ origin of platform fixed axes

$O_{c} \quad \mathrm{c} / \mathrm{m}$ of system $\left(\mathcal{S}=R+\sum_{i}\left(F_{i}+C_{i}\right)+B\right)$

$O_{c_{b}} \quad \mathrm{c} / \mathrm{m}$ of $R$

$O_{F_{i}} \quad$ origin of i-th ducted fan subsystem axes

$O_{G_{i}}$ origin of i-th CMG frame

Let $\omega_{s_{i}} \hat{a}_{i}, \Omega_{B} \hat{z}$, and $\dot{\eta}_{i} \hat{g}_{i}$, denote the relative angular velocities of the $i$ th fan, momentum wheel, and the gimbal system relative to platform, respectively. Additionally, let $\Omega_{i} \hat{s}_{i}$ ), denote the relative angular velocity of the $i$ th CMG wheel relative to its gimbal frame. For the CMG, assume that the center of mass of rotor is fixed in $F_{b}$, and that the gimbal axis, $\hat{g}$, is fixed in $F_{b}$, while the spin and output axes are defined as $\hat{s}$ and $\hat{o}$ respectively. For more details on CMGs, see for example [9]. We define the following frames:

$$
\begin{aligned}
F_{o} & \triangleq \text { bases for inertial frame, origin } O \\
F_{b} & \triangleq(\hat{x}, \hat{y}, \hat{z})^{T}=C F_{o} \text { bases for platform frame } \\
F_{F_{i}} & \triangleq C_{F_{i}} F_{b} \text { bases for i-th ducted fan frame } \\
F_{G_{i}}^{o} & \triangleq C_{G_{i}}^{o} F_{b}=\left(\hat{s}_{i}^{o}, \hat{g}_{i}, \hat{o}_{i}^{o}\right)^{T} \text { initial i-th gimbal frame } \\
F_{G_{i}} & \triangleq\left(\hat{s}_{i}, \hat{g}_{i}, \hat{o}_{i}\right)^{T} \text { i-th gimbal frame } \\
F_{G_{i}} & \triangleq C_{G_{i}}\left(\eta_{i}\right) F_{G_{i}}^{o}=C_{G_{i}} F_{b} \\
C_{G_{i}}\left(\eta_{i}\right) & \triangleq\left[\begin{array}{ccc}
\cos \eta_{i} & 0 & -\sin \eta_{i} \\
0 & 1 & 0 \\
\sin \eta_{i} & 0 & \cos \eta_{i}
\end{array}\right]
\end{aligned}
$$

In summary, we consider an 19 degrees of freedom NFTP model which includes the following:

3 positions of $O_{b}$ relative to $O$

3 rotations of $R$ relative to $F_{o}$

4 rotations of fan relative to $F_{b}$

1 rotation of $B$ relative to $F_{b}$

4 rotations of CMG gimbals relative to $F_{b}$

4 rotations of CMG wheels relative to $F_{G_{i}}$
The following momenta variables are used in describing the equations of motion:

$$
\begin{aligned}
\underline{p}= & m \underline{v}+\underline{\omega} \times \underline{c} \\
\underline{h}= & \stackrel{c}{\rightarrow} \times \underline{v}+\underline{J} \rightarrow \underline{\omega}+\sum_{i=1}^{4} I_{\gamma F_{i}}^{a} \omega_{s_{i}} \hat{a}_{i} \\
& +\sum_{i=1}^{4}\left(I_{C_{i}}^{g} \dot{\eta}_{i} \hat{g}_{i}+I_{W_{i}}^{a} \Omega_{i} \hat{s}_{i}\right)+I_{B}^{a} \Omega_{B} \hat{z} \\
h_{a_{i}}= & I_{F_{i}}^{a} \hat{a}_{i} \cdot \underline{\omega}+I_{\gamma F_{i}}^{a} \omega_{s_{i}}, \quad i=1, \ldots, 4 \\
h_{B z}= & I_{B}^{a} \hat{z} \cdot\left(\underline{\omega}+\Omega_{B} \hat{z}\right) \\
h_{W_{i 1}}= & I_{W_{i}}^{a}\left(\omega_{1}^{\eta_{i}}+\Omega_{i}\right), \quad i=1, \ldots, 4 \\
h_{C_{i 2}}= & I_{C_{i}}^{g}\left(\omega_{2}^{\eta_{i}}+\dot{\eta}_{i}\right), \quad i=1, \ldots, 4
\end{aligned}
$$

The vectors, $\underline{v}$ and $\underline{\omega}$ denote the platform translational and rotational inertial velocities, respectively, while $\vec{c}$ denotes the first moment of inertia about $O_{b}$ for the system having a mass $m$. The vectors, $\underline{p}$ and $\stackrel{h}{\rightarrow}$, denote the the translational and rotational momentum of the system, respectively. The angular velocity term, $\omega_{j}^{\eta_{i}}$ where $i=1, \ldots, 4$ and $j=1,2,3$ (corresponding to $\hat{s}_{i}, \hat{g}_{i}$, and $\hat{o}_{i}$ ), denotes the platform angular velocity components in the $j$ th axis of the $i$ the gimbal frame. In particular, if we represent the platform angular velocity in terms of body frame so that $\underline{\omega}=F_{b}^{T} \omega$, the above angular velocity terms can be expressed in matrix form as

$$
\omega_{1}^{\eta}=K \omega, \quad \omega_{2}^{\eta}=K_{o} \omega, \quad \omega_{3}^{\eta}=G \omega
$$

where

$$
K \triangleq\left[\begin{array}{c}
k\left(\eta_{1}\right) \\
k\left(\eta_{2}\right) \\
k\left(\eta_{3}\right) \\
k\left(\eta_{4}\right)
\end{array}\right], \quad K_{o} \triangleq\left[\begin{array}{c}
k_{o_{1}} \\
k_{o_{2}} \\
k_{o_{3}} \\
k_{o_{4}}
\end{array}\right], \quad G \triangleq\left[\begin{array}{c}
g\left(\eta_{1}\right) \\
g\left(\eta_{2}\right) \\
g\left(\eta_{3}\right) \\
g\left(\eta_{4}\right)
\end{array}\right]
$$

and for $i=1, \ldots, 4$

$$
\begin{aligned}
k\left(\eta_{i}\right) & \triangleq\left[\begin{array}{lll}
\cos \eta_{i} & 0 & -\sin \eta_{i}
\end{array}\right] C_{G_{i}}^{o} \\
k_{o_{i}} & \triangleq\left[\begin{array}{lll}
0 & 1 & 0
\end{array}\right] C_{G_{i}}^{o} \\
g\left(\eta_{i}\right) & \triangleq\left[\begin{array}{lll}
\sin \eta_{i} & 0 & \cos \eta_{i}
\end{array}\right] C_{G_{i}}^{o}
\end{aligned}
$$

For practical reasons, the rotational momentum is defined about the point $O_{b}$, which is the center of the platform frame, $F_{b}$, but need not be the center of mass of $\mathcal{S}$ due to practical limitations and/or not accurately known variable mass payload and its distribution during flight. Naturally additional complications arise due to the fact that reference point $O_{b}$ is neither the center of mass nor fixed to an inertial frame. The remaining variables, $h_{a_{i}}, h_{B z}, h_{W_{i 1}}, h_{C_{i 2}}$, are absolute rotational momenta vector components of the $i$ th rotating fan, momentum wheel, CMG wheel, and CMG system, along their respective primary spin axes (i.e. $\hat{a}_{i}, \hat{z}, \hat{s}_{i}, \hat{g}_{i}$ ). As reference points for the above momenta components, their respective center of masses are used. Of course the center of masses for 
these actuator subsystem hardware can be accurately determined and are not expected to vary during operation.

With the above choice of variables and notations, the equations of motion are summarized as follows (for details see $[8])$ :

$$
\begin{aligned}
& \underline{\dot{p}}=m \underline{g}+\sum_{i=1}^{4} \underline{T}_{i}+\stackrel{f}{\rightarrow e t}_{\text {jet }}+\stackrel{f}{\text { aero }} \\
& \stackrel{\dot{h}}{\rightarrow}=-\underline{v} \times \underline{p}+\underline{c} \times \underline{g}+\sum_{i=1}^{4}\left[\underline{\tau}_{i}+\underline{b}_{F_{i}} \times \underline{T}_{i}\right] \\
& +\stackrel{\tau}{\rightarrow}_{\text {jet }}+\stackrel{\tau}{\rightarrow}_{\text {aero }} \\
& \dot{h}_{a_{i}}=\tau_{F_{i}} \\
& \dot{h}_{B z}=\tau_{B z} \\
& \dot{h}_{W_{i 1}}=\tau_{\hat{s}_{i}} \\
& \dot{h}_{C_{i 2}}=-\omega_{3}^{\eta_{i}}\left[\left(I_{C_{i}}^{s}-I_{C_{i}}^{o}\right) \omega_{1}^{\eta_{i}}+I_{W_{i}}^{a} \Omega_{i}\right]+\tau_{\hat{g}_{i}}
\end{aligned}
$$

where $i=1, \ldots, 4$, i.e., 4 sets of ducted fans and single gimbal variable speed CMGs are assumed, but clearly any number can be used in these equations. The terms, $\stackrel{T}{\rightarrow}_{i} \triangleq \underline{T}_{W_{i}}+\underline{T}_{b_{i}}, \stackrel{\tau}{\rightarrow}_{i} \triangleq{\underset{\tau}{\rightarrow} W_{i}}_{\rightarrow}+{\stackrel{\tau}{b_{i}}}$, denote external aeropropulsive net thrust and torques on the system due to vector thrusting and differential throttling of the ducted fan and vane subsystem. The terms $\underset{\rightarrow \text { jet }}{\stackrel{f}{\rightarrow} \text { aero }}, \stackrel{\tau}{\rightarrow}_{j e t}$, and $\underset{\tau}{\rightarrow}$ aero denotes the aerodynamic loads on the system due to significant vehicle airspeed. In this paper, we focus on the subclass of problems where the vehicle is flying at low airspeeds, due to its particular difficulty in attitude stabilization. Hence we do not include these terms in this paper but is addressed in [8]. The terms $\tau_{\hat{s}_{i}}$ and $\tau_{\hat{g}_{i}}$ denotes the internal torques on the i-th CMG wheel due to the wheel motor, the internal torques on the i-th CMG subsystem due to the gimbal motor, respectively. The variable $\tau_{B_{z}}$ denotes the internal torque on the momentum wheel due to its motor, while $\tau_{F_{i}}$ denotes net torque on the i-th fan due to a combination of external fan rotational drag and internal fan motor torque, including back EMF effects.

\subsection{Aerodynamic and propulsive loads}

Figure 3 shows a set of frames used to conveniently describe various loads produced by engine thrust, vane deflections, and fan rotational drag.

\subsubsection{Net propulsive forces acting on $O_{F_{i}}$}

$$
\underline{T}_{i}\left(\omega_{s_{i}}, \theta_{i}\right)=F_{b}^{T} C_{F_{i}}^{T} T_{i}\left(\omega_{s_{i}}, \theta_{i}\right), \quad T_{i} \in \mathcal{R}^{3 \times 1}
$$

where

$$
T_{i}\left(\omega_{s_{i}}, \theta_{i}\right) \approx\left(\begin{array}{c}
c_{T_{x_{i}}} \\
c_{T_{y_{i}}} \\
c_{T_{z_{i}}}
\end{array}\right) \omega_{s_{i}}^{2}+\left(\begin{array}{c}
c_{x_{i}} \theta_{i} \\
c_{y_{i}} \theta_{i} \\
-c_{z_{i}} \theta_{i}^{2}
\end{array}\right) \omega_{s_{i}}^{2}:=e_{i}\left(\theta_{i}\right) \omega_{s_{i}}^{2}
$$

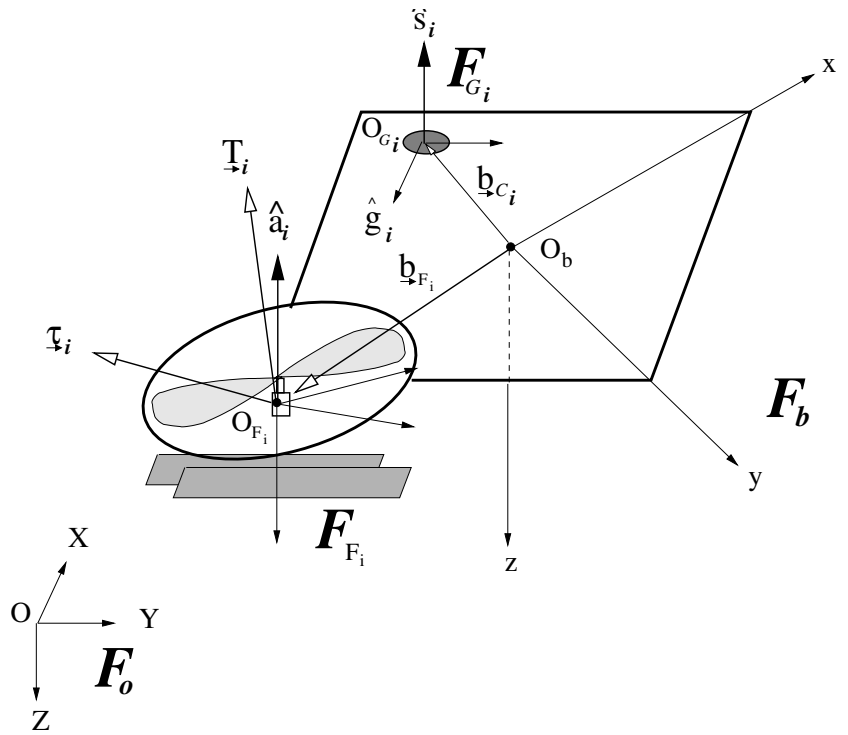

Figure 3: Reference frames for modeling loads produced by Fans, Vanes, and CMG.

\subsubsection{Net propulsive moments acting about $O_{F_{i}}$}

$$
\underset{\tau_{i}}{\rightarrow}\left(\omega_{s_{i}}, \theta_{i}\right)=F_{b}^{T} C_{F_{i}}^{T} \tau_{i}\left(\omega_{s_{i}}, \theta_{i}\right), \quad \tau_{i} \in \mathcal{R}^{3 \times 1}
$$

where

$$
\tau_{i}\left(\omega_{s_{i}}, \theta_{i}\right) \approx\left(\begin{array}{c}
c_{\tau_{x_{i}}} \\
c_{\tau_{y_{i}}} \\
c_{\tau_{z_{i}}}
\end{array}\right) \omega_{s_{i}}^{2}+\left(\begin{array}{c}
l_{x_{i}} \\
l_{y_{i}} \\
l_{z_{i}}
\end{array}\right) \theta_{i} \omega_{s_{i}}^{2}:=f_{i}\left(\theta_{i}\right) \omega_{s_{i}}^{2}
$$

\subsubsection{Rotational Drag on i-th Fan along $\hat{a}_{i}$}

$$
\hat{a}_{i} \cdot{\stackrel{\tau}{\rightarrow} W_{i}}=-\tau_{W_{i}}\left(\omega_{s_{i}}\right), \quad \tau_{W_{i}} \in \mathcal{R}
$$

where

$$
\tau_{W_{i}}\left(\omega_{s_{i}}\right) \approx c_{R_{i}} \omega_{s_{i}}^{2}
$$

Fan rotational drag only in $\hat{a}_{i}$ axis (i.e. excludes vane deflection effects).

\subsubsection{Torques on i-th Fan motor along $\hat{a}_{i}$}

$$
\hat{a}_{i} \cdot \underline{M}_{W_{i}} \triangleq \tau_{a_{i}}\left(\omega_{s_{i}}, \omega_{s_{i}}^{c m d}\right), \quad \tau_{a_{i}} \in \mathcal{R}, \quad i=1, \ldots, 4
$$

where

$$
\tau_{a_{i}}\left(\omega_{s_{i}}, \omega_{s_{i}}^{c m d}\right) \approx k_{E M F_{i}} \omega_{s_{i}}+k_{3} \omega_{s_{i}}^{c m d}
$$

The torque modeled in equation 24 is a combination of back EMF and armature current torques. The net torque on the fan system along spin axis due to rotational drag and motor

$$
\tau_{F_{i}} \triangleq-\tau_{W_{i}}\left(\omega_{s_{i}}\right)+\tau_{a_{i}}\left(\omega_{s_{i}}, \omega_{s_{i}}^{c m d}\right)
$$




\subsection{Scalar form}

For modeling and computational purposes it is more convenient to express the motion equations in scalar/matrix form. In particular, we choose to express vector quantities in terms of their components in $F_{b}, F_{F_{i}}$, and $F_{G_{i}}$ frames:

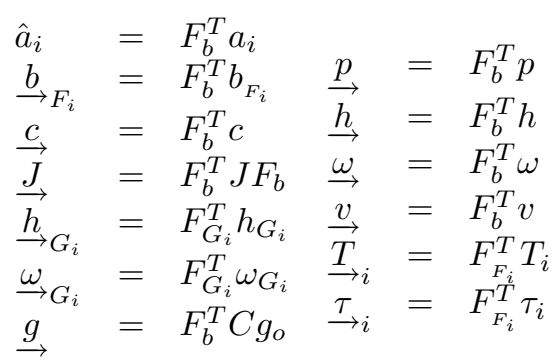

where all column matrix variables belong to $\mathcal{R}^{3 \times 1}$ except $J \in \mathcal{R}^{3 \times 3}$. These quantities include geometrical and inertial parameters of the vehicle that are constants in body frame, and state variables and forces and moments whose components can vary in body, gimbal, or fan frames.

The first order momenta equations for the total system are summarized as follows

$$
\begin{aligned}
\left\{\begin{array}{l}
\dot{p} \\
\dot{h}
\end{array}\right\}= & {\left[\begin{array}{cc}
-\omega^{\times} & 0 \\
-v^{\times} & -\omega^{\times}
\end{array}\right]\left\{\begin{array}{l}
p \\
h
\end{array}\right\}+\left[\begin{array}{c}
m I_{3 \times 3} \\
c^{\times}
\end{array}\right] C g_{o} } \\
& +\left[\begin{array}{cc}
C_{F}^{T} & 0 \\
C_{b F} & C_{F}^{T}
\end{array}\right]\left\{\begin{array}{c}
T\left(\omega_{s}, \theta\right) \\
\tau\left(\omega_{s}, \theta\right)
\end{array}\right\}
\end{aligned}
$$

and for ducted fan along spin axis are

$$
\dot{h}_{a}=\tau_{F}\left(\omega_{s}, \omega_{s}^{c m d}\right)
$$

and for momentum wheel along spin axis, $\hat{z}$

$$
\dot{h}_{B z}=\tau_{B z}\left(\Omega_{B}, \Omega_{B}^{c m d}\right)
$$

and for CMGs along spin and gimbal axes are

$$
\left\{\begin{array}{c}
\dot{h}_{W \cdot 1} \\
\dot{h}_{C \cdot 2}
\end{array}\right\}=\left\{\begin{array}{c}
\tau_{\hat{s}} \\
-\operatorname{diag}(G \omega)\left[\left(I_{C}^{s}-I_{C}^{o}\right) K \omega+I_{W}^{a} \Omega\right]+\tau_{\hat{g}}
\end{array}\right\}
$$

The momenta is related to the velocities as follows

$$
\left\{\begin{array}{c}
p \\
h \\
h_{a} \\
h_{B_{z}} \\
h_{W \cdot 1} \\
h_{C \cdot 2}
\end{array}\right\}=\left[\begin{array}{c|c}
M & \Phi(\eta)^{T} \\
\hline \Phi(\eta) & \Psi
\end{array}\right]\left\{\begin{array}{c}
v \\
\omega \\
\omega_{s} \\
\Omega_{B} \\
\Omega \\
\dot{\eta}
\end{array}\right\}
$$

where $M \triangleq\left[\begin{array}{cc}m I_{3 \times 3} & -c^{\times} \\ c^{\times} & J\end{array}\right], \quad \Phi(\eta) \triangleq$
$\left[\begin{array}{cc}0_{4 \times 3} & I_{F}^{a} A^{T} \\ 0_{1 \times 3} & \left(00 I_{B}^{a}\right) \\ 0_{4 \times 3} & I_{W}^{a} K \\ 0_{4 \times 3} & I_{C}^{g} K_{o}\end{array}\right]$, and $\Psi \triangleq \operatorname{diag}\left(I_{\gamma F}^{a}, I_{B}^{a}, I_{W}^{a}, I_{C}^{g}\right)$. In the above choice of dynamical equation coordinates, the inertial attitude of the platform (i.e. $C$ ) enters through the effects of gravity. Its inadvertent effects on rotation is due to the limited knowledge in the exact location of the system center of gravity. If the center of gravity of the whole system is known, then, $O_{b}$ can be chosen as such and the rotational and translational motions uncouple.

In the above equations, the superscript $(\cdot)^{\times}$denotes the skew-symmetric matrix associated with vector cross products, for example, $\omega^{\times} \triangleq\left[\begin{array}{ccc}0 & -\omega_{3} & \omega_{2} \\ \omega_{3} & 0 & -\omega_{1} \\ -\omega_{2} & \omega_{1} & 0\end{array}\right]$. For the definition of augmented states and variables including $\omega_{s}, h_{a}, \Omega, \eta, h_{W_{\cdot 1}}, h_{C \cdot 2}, T, \tau, \tau_{W}, \tau_{a}, \tau_{\hat{s}}, \tau_{\hat{g}}, \tau_{F}$, and augmented parameters including $C_{F}, C_{b F}, A, I_{M}^{a}, I_{P}^{a}, I_{F}^{a}, I_{\gamma F}^{a}, I_{W}^{a}, I_{C}^{g}$, see [8] for details.

For kinematics, we denote the position of the origin, $O_{b}$, of the platform-fixed frame, $F_{b}$, with respect to inertial frame, $F_{o}$ as $\underline{R}_{O_{b}}=F_{o}^{T} \xi$ so that the inertial velocity of $O_{b}$ is $\underline{v}=F_{b}^{T} v=F_{o}^{T} \dot{\xi}$, and the inertial velocity components in $F_{o}$ frame can be written as $\dot{\xi}=C(\beta)^{T} v$ where we choose to parameterize the direction cosine matrix relating $F_{o}$ to $F_{b}$ by Euler Parameters, $\beta:=\left(\beta_{0}, \beta_{1}, \beta_{2}, \beta_{3}\right)^{T} \in \mathcal{R}^{4 \times 1}$. For the form of this direction cosine matrix parameterization and the time derivative of these Euler parameters, see for example [10].

\section{Flight Dynamics \& Control}

\section{$3.1 \quad$ Trim}

We initially consider the flight dynamics as referenced to trim conditions which we define as attitude hold while moving at constant translational speeds. This of course is suited only for cruise and hovering flight segments. The trim conditions are found by calculating any combinations of four vane angles and four fan speeds necessary to hold a given desired attitude, at a constant translational velocity. With variable speed CMGs we choose a nominal wheel speeds and zero gimbal rotation rate as the trim value. In summary, the trim conditions are defined by setting all momenta state derivatives, given in equations 26 to 29 , to zero. In addition, we consider $\hat{\omega}=0_{3 \times 1}$ to hold platform attitude to $\hat{\beta}$.

Assuming the above conditions, the first order momenta equations for trim reduces to a coupled set of algebraic equations [8]. We make the following observations from the trim conditions:

- Because $\hat{\omega}=0, \hat{p}=m \hat{v}$, i.e. at trim, the linear momenta do not affect rotational equilibrium, i.e., $\hat{v}^{\times} \hat{p}=0$.

- For a given steady or near trim flight condition, a combination of vector thrusting via fan speeds, $\hat{\omega}_{s}$, and vane angle deflections, $\hat{\theta}$, are used to satisfy trim conditions. In the case when the vehicle is required to trim at airspeeds that result in significant vehicle 
aerodynamic loads, as described in equations 11 and 12 , the above trim conditions can be slightly modified.

- For the ducted fan trim $\dot{\hat{h}}_{a}=0$, the fan control moments, $\hat{\tau}_{a}$, exactly balance the fan rotational drag, $\hat{\tau}_{W}$ as given in equation (25). The trim command can be computed once the required fan speed $\hat{\omega}_{s}$ is known from system momenta equilibrium equations.

- For the momentum wheel trim, $\dot{h}_{B_{z}}=0$, the wheel control moments, $\tau_{B}^{\text {motor }}$ exactly balances the wheel friction, $\tau_{B}^{\text {friction }}$. The desired level of bias momentum for the overall system defines the momentum wheel trim speed, $\hat{\Omega}_{B}$, which will determine its corresponding command, $\hat{\Omega}_{B}^{c m d}$.

- For each CMG wheel trim where $\hat{\dot{h}}_{W \cdot 1}=0$, the CMG wheel motor must exactly balance the CMG wheel friction at a chosen trim speed $\hat{\Omega}$.

- For each CMG gimbal system trim where $\hat{\dot{h}}_{C_{.2}}=0$, the CMG gimbal motor torque must be zero corresponding to gimbal rate trim, $\hat{\dot{\eta}}=0$.

The trim conditions can be expressed only in terms of the trim angles, $\hat{\theta}$, and fan angular velocities, $\hat{\omega}_{s}$, by using the vector thrusting models as given in equations (17) to (24) for the forces and moments produced by fan rotation and vane deflections. These key subset of trim conditions can be rearraged to the following compact form:

$$
M(\hat{\theta}) \hat{\omega}_{s}^{2}+\phi(\hat{\beta})=0_{6 \times 1}
$$

where

$$
\begin{aligned}
M(\hat{\theta}) & :=\left[\begin{array}{c}
C_{F}^{T} \\
\frac{1}{b_{o}} C_{b F}
\end{array}\right] E(\hat{\theta})+\left[\begin{array}{c}
0 \\
\frac{1}{b_{o}} C_{F}^{T}
\end{array}\right] F(\hat{\theta}) \\
\phi(\hat{\beta}) & :=\left[\begin{array}{c}
m I_{3 \times 3} \\
\frac{1}{b_{o}} c^{\times}
\end{array}\right] C(\hat{\beta}) g_{o}
\end{aligned}
$$

define the geometry, kinematics, mass properties, secondary trim actuators, and vehicle aerodynamics. The scalar constant $b_{o}$ meters is a reference moment arm to help scale the combined force-moment equations to force units, which helps in the selection of a treshold numerical value for defining equilibrium.

For any given set of variables, $\left(\hat{\beta}, \hat{\theta}, \hat{\omega}_{s}, v\right)$, that satisfies the vector thrusting trim equations (31), one can deduce the corresponding ducted fan speed trim command $\omega_{s_{i}}^{c m d}$. The remaining trim commands, momentum wheel speed $\hat{\Omega}_{B}^{c m d}$, CMG wheel speed $\hat{\Omega}^{c m d}$, and CMG gimbal rate $\hat{\dot{\eta}}^{\text {cmd }}$, will clearly depend on their corresponding desired trim conditions $\left(\hat{\Omega}_{B}, \hat{\Omega}, \hat{\dot{\eta}}=0\right)$, and their corresponding motor dynamics. Note that these details involve motor commands to regulate and track armature rotation speeds, and involve technology that is well understood and developed.
Proposition 1 (Trimability) Given a platform attitude, $\hat{\beta}$, the system defined by equations (26)-(29) is trimmable, using vector thrusting (using a combination of control vane deflections, $\hat{\theta}$, fan thrusts with fan rotation speeds $\hat{\omega}_{s}$, and reaction jets), if and only if there exists $\hat{\theta}$ such that $\phi(\hat{\beta}) \in \operatorname{Range}[M(\hat{\theta})]$.

If the above trim conditions are met for $\hat{\beta}$ and $\hat{\theta}$, then, the corresponding set of all possible fan speed combinations can be parameterized as follows:

$$
\hat{\omega}_{s}^{2}=-M(\hat{\theta})^{+} \phi(\hat{\beta})+\psi \quad \text { where } \psi \in \operatorname{Null}[M(\hat{\theta})]
$$

\section{Proposition 2 (Numerical Criteria for Trim)}

Given a platform attitude, $\hat{\beta}$, the system defined by equations (26)-(29) is trimmable, using vector thrusting if the following least squares equation error is satisfied:

$$
\|\varepsilon(\hat{\beta}, \hat{\theta})\|_{2}:=\left\|\left[I-M(\hat{\theta}) M(\hat{\theta})^{+}\right] \phi(\hat{\beta})\right\|_{2} \leq \epsilon
$$

The superscript symbol in $(\cdot)^{+}$denotes its pseudo-inverse. Note that the treshold for zero equation error denoted as $\epsilon$ should reflect practical limitations including (i) numerical error, in the order of machine epsilon, (ii) accuracy limitation in the actuation and measurement of vane angles, (iii)accuracy limitation in the fan thrust response to command, including integer command resolution, and (iv) sensor accuracy limitaion in a high quality accelerometer. These factors are used to define an engineering treshold of "trim" for the NFTP during low airspeed operations. Since trim angles and speeds are non-unique if they exists, we so choose the "smoothest" combination, see [8].

\subsection{Gyric Stability about trim}

Figure 4 shows the perturbed dynamics about trim. In

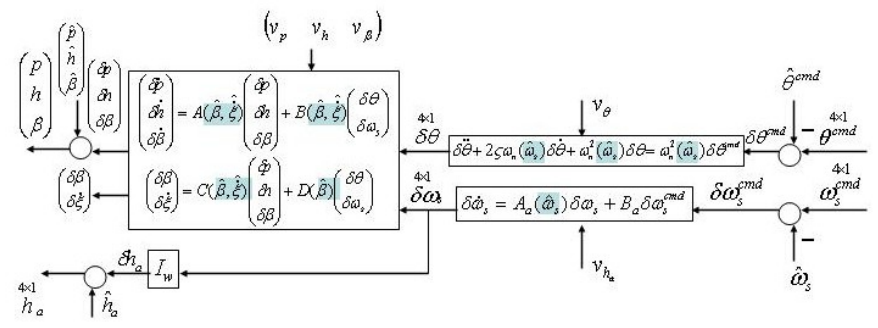

Figure 4: Perturbed dynamics about trim.

general, the open loop dynamics involves complex perturbed response due to gyroscopic coupling in the rotation axes and the coupling with translational degrees of freedom (in addition to coning due to axis misalignments and translational-rotational coupling due to off CG effects). Hence for the sake of analytical tractability, we simplify by assuming a perfect knowledge of system CG location and the orientation of the system principal axes. With these assumptions, the tenth-order system characteristic equation for the linearly perturbed system shown 
in Figure 4 can be written and solved explicitly. It turns out that the only non-zero eigenvalues have the form

$$
\lambda_{9,10}= \pm j \lambda_{o}, \quad \text { where } \lambda_{o}:=\frac{\left|h_{B}\right|}{\sqrt{J_{1} J_{2}}}
$$

Physically, this complex conjugate pair corresponds to the gyroscopic coupling in the pitch and roll axes. This oscillation or wobbling frequency given in equation 36 is analogous to the "precession frequency" in a dual-spin spacecraft (a gyrosat with a nonspinning carrier) as derived for example in Chapter 6 of [7]. Clearly, this wobbling frequency increases linearly with the level of bias momentum and is inversely proportional to the rotational inertia of the vehicle. It can be shown that the transfer function matrix from external torques $\left(\nu_{1}, \nu_{2}, \nu_{3}\right)$ to small angular responses in roll, pitch, and yaw has the form

$$
\left\{\begin{array}{l}
\delta \beta_{1} \\
\delta \beta_{2} \\
\delta \beta_{3}
\end{array}\right\}=\left[\begin{array}{c|c}
\frac{1}{s} G\left(s ; h_{B}, J_{1}, J_{2}\right) & 0_{2 \times 1} \\
\hline 0_{1 \times 2} & \frac{1}{s^{2} J_{3}}
\end{array}\right]\left\{\begin{array}{c}
\nu_{1} \\
\nu_{2} \\
\nu_{3}
\end{array}\right\}
$$

where the roll-pitch transfer matrix is

$$
G\left(s ; h_{B}, J_{1}, J_{2}\right) \triangleq \frac{1}{s^{2}+\lambda_{o}^{2}}\left[\begin{array}{cc}
\frac{s}{J_{1}} & -\frac{h_{B}}{J_{1} J_{2}} \\
\frac{h_{B}}{J_{1} J_{2}} & \frac{s}{J_{2}}
\end{array}\right]
$$

Figure 5 shows the roll angle frequency response from disturbance torques in the roll and pitch axes. Notice that the linear yaw response depends only on the yaw disturbance torque and is independent of roll and pitch motion. The main point is that bias momentum can significantly improve attitude stability by reducing the response to disturbances in roll and pitch. Furthermore, as the level of bias momentum increases, the roll response to roll disturbances decreases more rapidly than to pitch disturbance torque. Alternately, the dynamically uncoupled system at zero bias momentum transforms to a system whose characteristic dynamics is gyrically dominated.
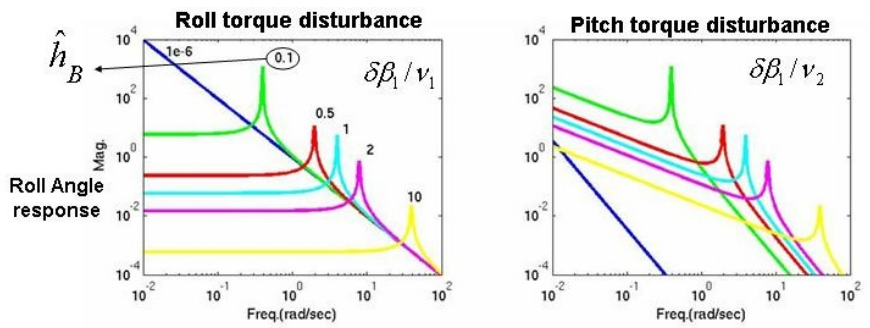

Figure 5: Open loop frequency response in roll and pitch angles at different levels of bias momentum.

\subsection{Bias Momentum Sizing}

Consider the rotational dynamics of a generic dual-spin system with principal rotational inertias $\left(J_{1}, J_{2}, J_{2}\right)$ driven by disturbance torques $\left(\tau_{1}, \tau_{2}\right.$, and $\left.\tau_{3}\right)$ in all three axes. Suppose the main body rotates with angular velocity components in body coordinates denoted by $\left(\omega_{1}, \omega_{2}\right.$, and $\left.\omega_{3}\right)$ while the momentum wheel spins about the vertical (3-axis) at a fixed rate relative to the main body to produce a constant bias momentum of $h_{B}$. In addition, suppose the bias momentum in the wheel "dominates" in the following sense

$$
\left|h_{B}\right| \gg \max \left(\left|\left(J_{3}-J_{2}\right) \omega_{3}\right|,\left|\left(J_{3}-J_{1}\right) \omega_{3}\right|\right)
$$

Then, the dynamics of this generic dual spin system can be approximated by

$$
\begin{aligned}
& J_{1} \dot{\omega}_{1} \approx-\omega_{2} h_{B}+\tau_{1} \\
& J_{2} \dot{\omega}_{2} \approx \omega_{1} h_{B}+\tau_{2} \\
& J_{3} \dot{\omega}_{3}=\left(J_{2}-J_{1}\right) \omega_{1} \omega_{2}+\tau_{3}
\end{aligned}
$$

These equations are not limited to "small" angular motion (associated with linearizations) but rather to the dominance of the bias momentum, as defined in equation 39. Although the yaw dynamics in equation 42 is nonlinear, the roll-pitch dynamics in equations 40 and 41 are linear. In fact this transfer function matrix for roll-pitch dynamics is identical (except $1 / s$ term) to the dual-spin linearized roll-pitch dynamical equations 38 .

For this generic dual spin system, we consider a bias momentum sizing approach based on the angular velocity response of the main body to a set of disturbances. To obtain an analytical expression for roll-pitch response to random disturbance torques, we make some mild assumptions on these unknown disturbances. Suppose the power spectral densities of the disturbance torques caused by unknown random turbulence, $\tau_{1}$, and $\tau_{2}$, with a common bandwidth of $B W_{\tau}$, can be modeled as

$$
V(j \nu) \triangleq \operatorname{diag}\left(\rho_{\tau_{1}}, \rho_{\tau_{2}}\right), \quad \forall \nu<B W_{\tau}
$$

where $\nu$ denotes the dummy frequency parameter. It can be shown that [8] the mean square response of the roll and pitch angular velocities can be expressed as follows

$$
\mathcal{E}\left[\omega_{1}^{2}+\omega_{2}^{2}\right] \approx \frac{\sqrt{2} \max \left(\rho_{\tau_{1}}, \rho_{\tau_{2}}\right)}{\sqrt{J_{1} J_{2}}\left|h_{B}\right|}
$$

where the above estimate applies for responses at lower frequencies $\nu<\min \left(B W_{\tau}, \lambda_{o}\right)$. Figure 6 shows the effect of scaling bias momentum on the worst case angular velocity response in the roll and pitch axis, in terms of the maximum singular value frequency response. It follows then that if we want the RMS angular velocity response to be bounded by a certain maximum desirable value, then the bias momentum can be sized to satisfy an inequality based on equation 44 . In addition to the above requirement on the RMS response of the angular velocities, the sizing of the bias momentum should be such that the precession frequency lies outside the bandwidth of the disturbance, $B W_{\tau}$. This bandwidth requirement can be satisfied if we choose bias momentum level to satisfy, based on equation 36, the following condition:

$$
\lambda_{o}:=\frac{\left|h_{B}\right|}{\sqrt{J_{1} J_{2}}}>B W_{\tau}
$$




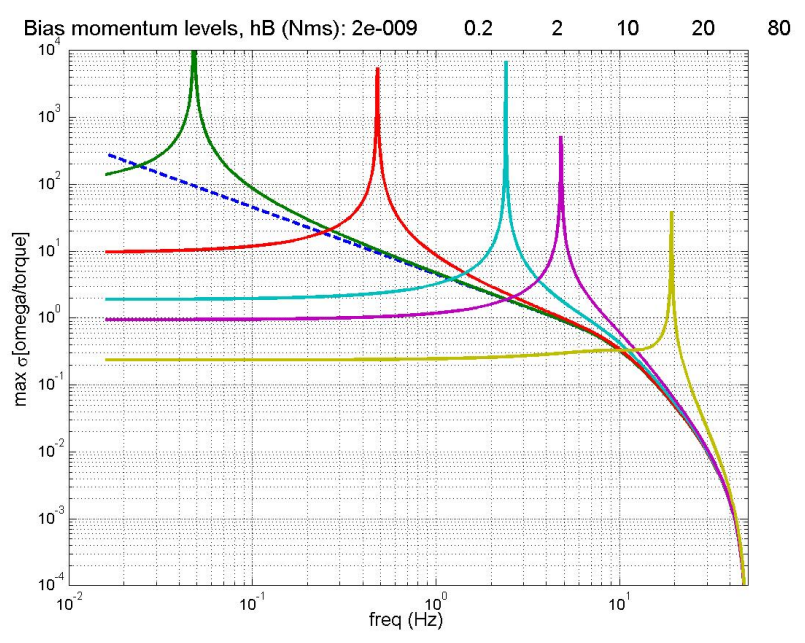

Figure 6: Maximum singular value frequency response in roll and pitch anglular velocities for sizing bias momenta.

\subsection{Control system framework}

Figure 7 shows a flight dynamics and control schematic for a general NFTP vehicle with the use of both bias momentum wheel and a set of variable speed CMG's. The vector thrusting commands include four fan speeds

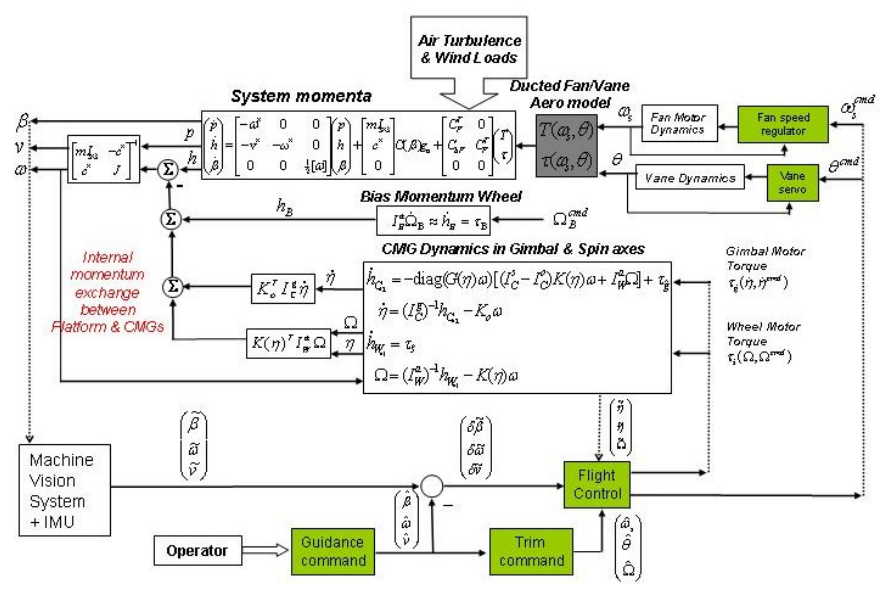

Figure 7: Flight dynamics and control schematic.

$\left(\omega_{s}^{c m d}\right)$ and four vane angles $\left(\theta^{c m d}\right)$ to control platform attitude $(\beta)$, and velocities ( $v$ and $\omega)$. These vector thrusting commands for the fans and vane angles are primarily used for generating trim conditions and for open loop command tracking. For the general configuration with CMGs, a secondary but nevertheless crucial function of the vector thrusting commands will be to stabilize the CMGs so that their saturation tendencies will be mitigated. To improve performance, fan speed regulators and vane servos are used.

Notice that the Ducted Fan/Vane aerodynamics and gravity directly influence system momenta $p, h$, while the CMG Dynamics has no influence on system momenta.
Rather, the CMGs internally redistribute angular momentum to control the platform angular velocity $\omega$ and its attitude $\beta$. A key advantage is that the CMG can generate internal control torques to effectively and very reliably redistribute angular momentum to control the platform, independently of Ducted Fan/Vane aerodynamics. This of course mitigates the uncertain but significant effects of unsteady aerodynamics induced by rapid control surface motions for attitude stabilization. This property of this control approach will particularly benefit smaller vehicles during hovering or operations at low airspeeds under significant wind turbulence because they will respond rapidly due to their smaller rotational inertias and will therefore require control effectors that can reliably generate control forces and moments at higher bandwidth for attitude stabilization.

While the bias momentum wheel is regulated to a certain constant speed, $\Omega_{B}$, to provide directional stability in open loop, the CMG subsystem will consist of closed loop torque commands to gimbal and wheel motors for variable speed CMGs. These commands will be generated by a control law which will integrate the CMGs with the vector thrusting control system for a given momentum wheel augmented platform. In particular, an approach with extends the steering laws outlined in [16], [17], and [15] to account for factors including, trim requirements, gravity effects, significant persistent air turbulence, translational coupling dynamics, and CMG desaturation using ducted fan based vector thrusting has been developed.

An alternate control viewpoint is based on the linear dynamics in Figure 4. This perturbed dynamical system can be viewed as an linear parameter varying (LPV) system for control law design wherein the scheduled parameters may include the trim parameters such as attitude and translational velocity. This viewpoint is particularly attractive because the control law analysis and synthesis is well understood and technologically viable (see for example [11],[12],[13],[14]). For further details on both control law approaches, see [8].

\section{$4 \quad$ Flying Test Platform}

\subsection{System overview}

The attitude control approach described above is tested using the vehicle pictured in Figure 8 as a CAD model. The vehicle is a platform levitated by four ducted fans arranged symmetrically about the $z$-body axis. Each fan duct is equipped with a parallel pair of thrust vectoring vanes, downstream from its fan. Each fan and pair of vanes is individually commanded by the control system. The $z$-body axis angular momentum bias is provided by a momentum wheel, powered separately from the levitation fans, and can be seen in the Figure at the center of the platform. Although the simplest and lightest momentum biasing mechanism is provided by augmenting the $z$-body 
axis moment of inertia of the propulsion system, momentum wheel is separately mechnized in order to facilitate tests in which different magnitudes of momentum bias are explored. For initial testing, the vehicle will operate without CMGs or reaction wheels.

In its initial configuration, the fan motors and momentum wheel motor are Aveox F27-F5B, and JR DS8411 are the vane servos which provides $300 \mathrm{deg} / \mathrm{s}$ maximum deflection rate. Each of the four levitation fan motors draw two kilowatts at full thrust. Because of this, the vehicle will be fed power from an overhead tether whose tension is controlled to offset its weight. The tether will mount to the platform at the vehicle's center of mass, to minimize tether-induced moment disturbances during flight. The dynamics of the tether are ignored in the next Section's simulation model. The attitude stabilization software is hosted on a PC104 computer that is affixed to the platform, and which communicates with a ground-based dSpace real-time control system. This latter is used to generate trim and guidance commands. The platform hosts an IMU model MIDG II, which is supplemented by a machine vision subsystem that gives Earth-fixed position and attitude. More details of this system are given in [8].

Each fan produces roughly 10 lbf of thrust at full power, which is more than adequate to levitate the gross platform mass of approximately $29 \mathrm{lbm}$. The moments of inertia (in $\mathrm{Kg}_{-} \mathrm{M}^{2}$ units) for this vehicle are $I_{x x}=0.59$, $I_{y y}=0.58, I_{z z}=1.15$, and $I_{i j} \approx 0, \forall i \neq j$.

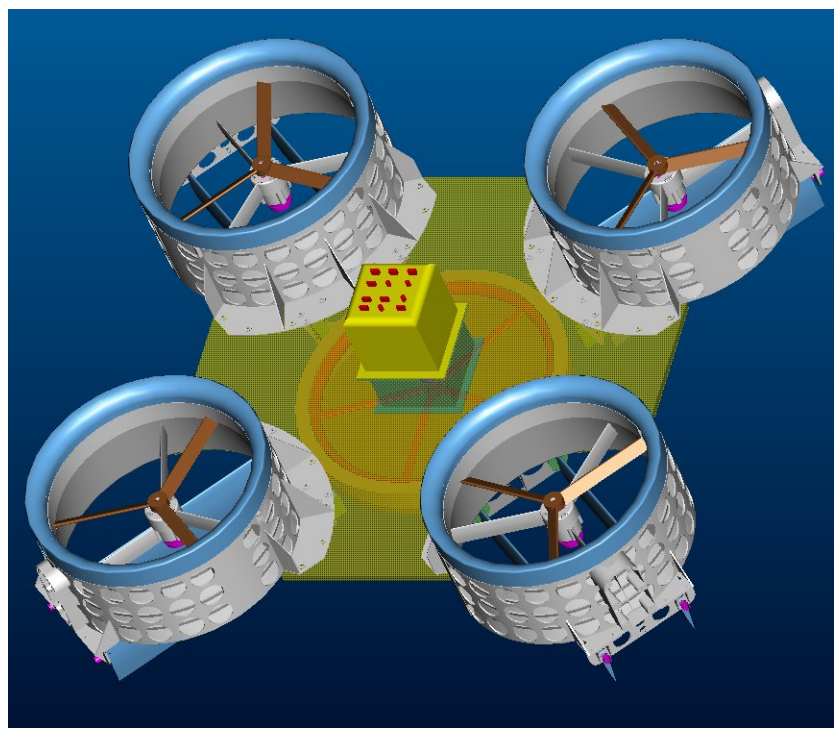

Figure 8: The NASA Flying Test Platform.

\subsection{Bench Testing}

In order to generate control design models and perform detail dynamical simulations which are physically relevant, we first characterize by parameterizing the dynamics of the subsystems and total system from first princi- ples and then use a series of bench tests to determine these parameters from measured data. Specific necessary tasks to this end includes design and fabrication of specific test configurations, instrumentation, calibration, followed by model development and validation through estimation of parameters including noise levels and its associated filters.

Figure 9 shows the bench test configuration for an individual ducted fan and vane system. The focus in this bench test involves characterizing (i) Fan motor and vane dynamics, and (ii) Fan and vane aerodynamics. The former basically involves the development of models to characterize fan speed and vane angle from their commands, while the latter modeling task can be anywhere from almost trivial to near impossible if the unsteady aero effects are to be included with any level of confidence. Together they determine the actual net forces and torques generated by control commands for fan speeds and vane deflection angles.

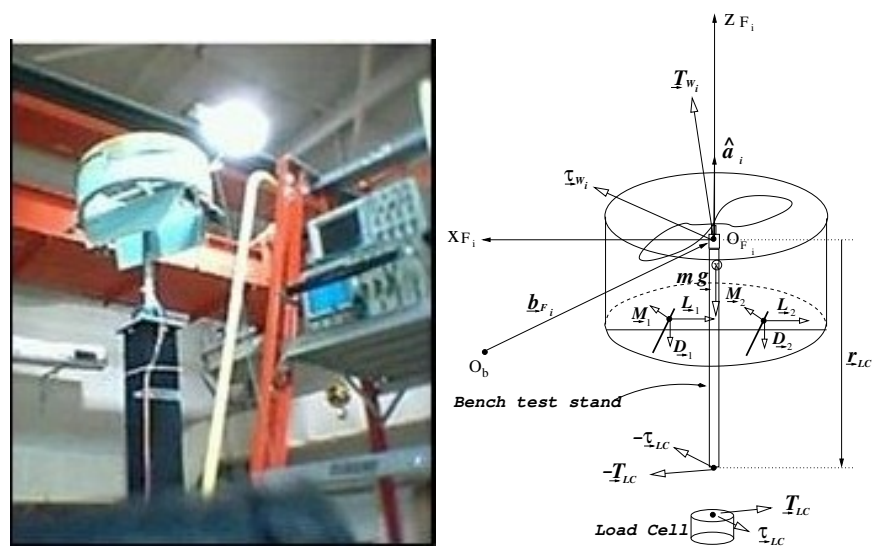

Figure 9: Bench test configurations and modeling.

Initially we consider a static aero model for the control forces and torques generated from fan speed and vane angle. For example, consider determining a steady aero model of net forces due to fan and vane. Figure 10 shows the measured net forces as a function of fan speed, for a fixed vane angle, while Figure 11 shows normalized measured vertical forces as a function of van angle, at constant fan speeds. Figure 12 illustrates the measured unsteady effects in the vertical force component with time varying fan speeds with fixed vane angle. The $1-\sigma$ variation of 0.1 pound thrust in a single fan is not insignificant for this subscale platform, especially considering that we have not included additional factors such as vane effects and implicit bandwidth limitations in the fan motor.

The above sample data were obtained experimentally, by testing the components in a ducted fan configuration, displayed in Figure 9. Static thrust vector force components were obtained from these tests, and are provided in [8]. Unsteady flow forces which are difficult to characterize are not quantified, and do not appear in the simulation model described in the next Section. 

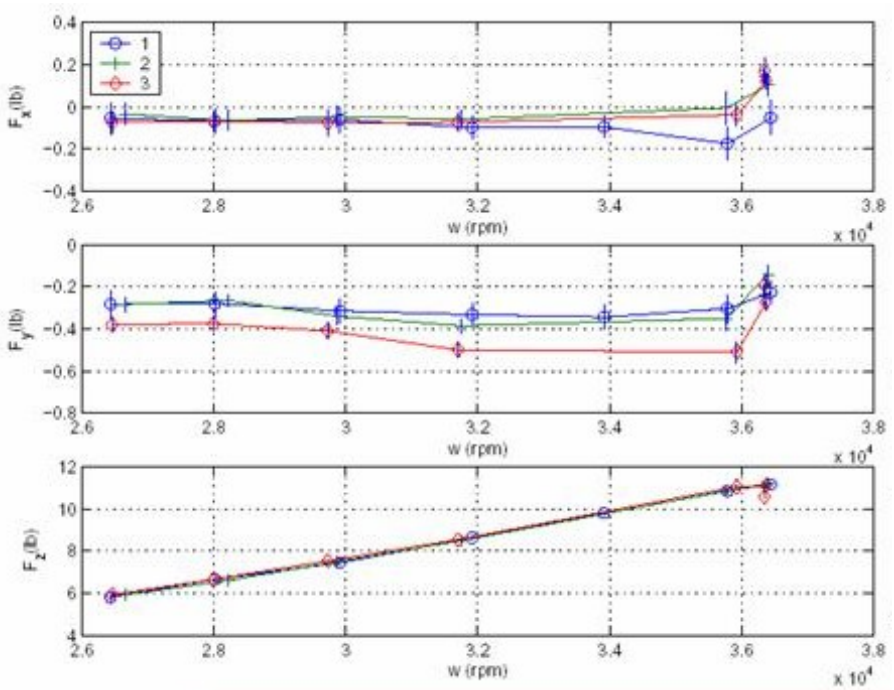

Figure 10: Measured forces with fan speeds, at fixed 0 degree vane angle.

\subsection{Simulation}

In this section we present simulation results based on full nonlinear models that are constructed from first principles, bench test data refined model parameters, sampled and quantized measurements, and saturation limits on actuators. In particular, the dynamical models for the Ducted fan/vane, whose development are based on bench tests, includes asymmetrical effects which are difficult to visualize due to their 3-dimensional characteristics. This MATLAB- Simulink simulation model also incorporates models of servo dynamics that regulate fan speeds and vane angles.

In all simulation cases which follows, the vanes and the fan speeds are actively used for stabilization and control which reflects the current state-of-the art approach which is vector and differential thrusting, albeit, RCquality indoor lab experiments. Initially, we focus our simulation on the effects of bias momentum in the stabilization and control performance of our flying test platform and do not include CMGs. We highlight these effects by comparing a system with significant level of bias momentum (BM cases) with a corresponding system with no bias momentum (NMB). A difference in our simulation studies is to independently control all four fan speeds and their respective vane angles, as opposed to constraining it to work in pairs and collectively, as in conventional helicopter control [18],[19] or practically all VTOL flying platforms [1].

\subsubsection{Crosswind and turbulence model}

To simulate wind disturbances during hovering or low airspeed operations, crosswinds are assumed which impinges on a vertical cylinder attached to the upper part of the platform. The resulting aerodynamic drag on this cylin-

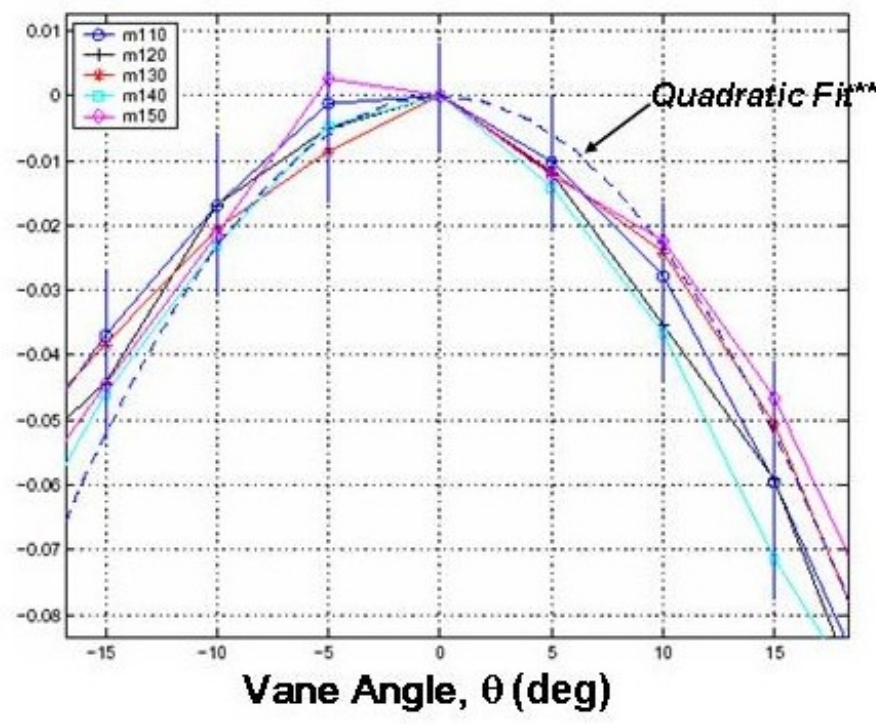

Figure 11: Measured and quadratic fit vertical force with vane angles, at different fan speeds.

drical column is used to simulate the crosswind and turbulence on the vehicle. The resulting disturbance model consists of forces and torques in the pitch and roll axis. Two sets of winds are simulated, namely, "light" winds, and "strong" winds. The "light" winds simulate "Calm to Gentle Breeze" with sample mean wind speeds of 7 Knots and standard deviation of 5 . However for "strong" winds, intended to simulate "Calm to Strong Breeze/Near Gale", the simulated sample mean wind speed is 15 Knots with a standard deviation of 18, as seen in Figure 13.

\subsubsection{Controller Law Synthesis}

Based on LQG control theory, feedback controllers are designed to stabilize and hold the platform attitude and also track the translational velocity and angular velocity commands. In this simulated design study, feedback controllers for the BM and NBM cases are synthesized based on the linearized model about hovering trim, which is identical to the trim conditions at a given translational velocity if aerodynamics due to significant airspeeds are not included, as assumed in this study. Note that the trim conditions are necessarily different for both cases since aero-resistance fan drag torques in the case must be statically canceled by vane-deflected forces.

The augmented linearized NFTP model with fan and servo models has 21 states consisting of 3 attitude parameters, 3 linear momentum, 3 angular momentum, 8 fan motor states, and 4 servo motor states. The 8 fan motor states includes the PI controllers which regulate each fan speed. The outputs of the augmented model are translational and angular velocities, both expressed in vehicle fixed coordinates. For a fair comparison between BM and NBM cases, the same LQG weights were used in the controller design 


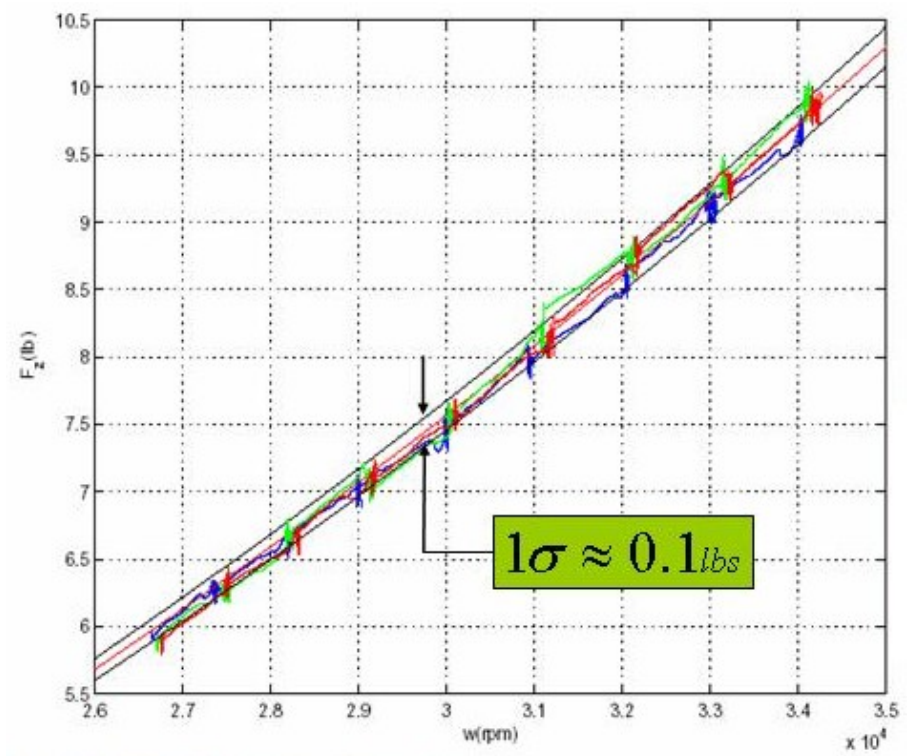

Figure 12: Measured uncertainty in the vertical force with time varying fan speeds at a fixed vane angle.

Figure 14 shows the tradeoff between performance cost and control effort for a parameterized set of LQG controllers undergoing gust response during hover over a period of time $T_{\text {final }}$. Specifically, the performance cost in terms of attitude and position errors are

$$
J(t) \triangleq \int_{0}^{t} \sum_{i=1: 3}^{3}\left[\left(\beta_{i}-\hat{\beta}_{i}\right)^{2}+\left(\xi_{i}-\hat{\xi}_{i}\right)^{2}\right] d t
$$

and the control effort is defined by a cumulative control effort (CCA) function as follows

$$
C C A(t) \triangleq \int_{0}^{t}\left(\dot{\theta}_{1}^{2}+\dot{\theta}_{2}^{2}+\dot{\theta}_{3}^{2}+\dot{\theta}_{4}^{2}\right) d t
$$

The abscissa and ordinate in Figure 14 represents $C C A\left(T_{\text {final }}\right)$ and $J\left(T_{\text {final }}\right)$ respectively. In addition to quantifying the level of control activity, the above control effort is intended to capture the level of control vane activity, which is seen as the primary source of control surface induced unsteady aerodynamics due to its vector thrusting role. Albeit limited, this tradeoff study indicates that controllers are significantly more effective for a system endowed with significant levels of bias momentum (asterisk line) in terms of actuator effort necessary for a given level of performance cost. The figure also shows the effects of a hypothetical model of unsteady aero dependence on actuator effort [8], namely, the expectation that controlled performance can inadvertently degrade with overly active control surface activity as mostly seen in the NBM case (diamond line). However, in the following simulations, this hypothetical model of controller induced unsteady aerodynamics will not be included.
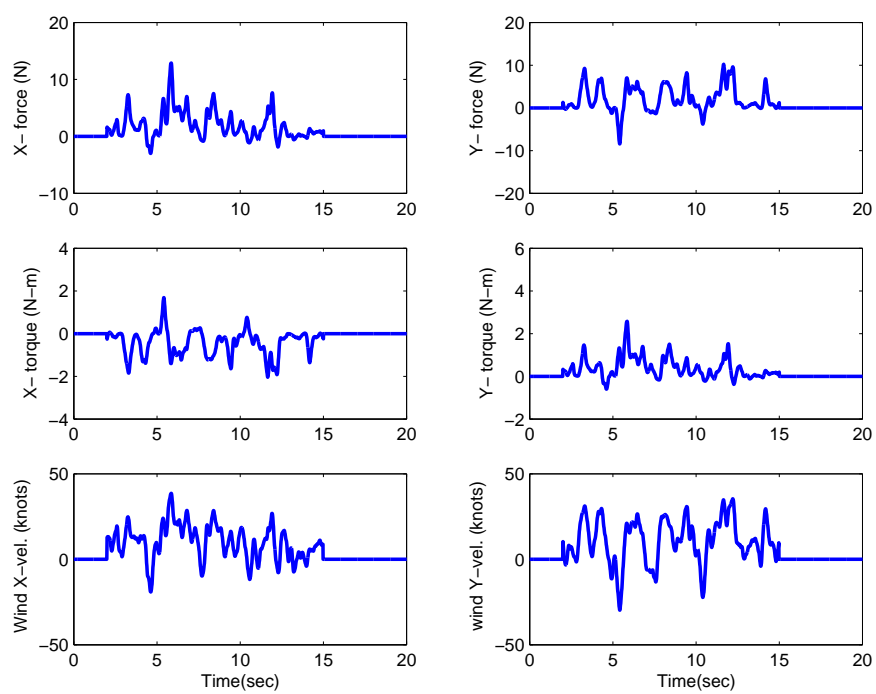

Figure 13: Simulated strong wind.

\subsubsection{Hovering}

Open Loop Response to Light Winds. The openloop attitude responses of the initially trimmed system for the two cases are shown in Figure 15. In the NBM case, the vehicle rapidly loses orientation and goes unstable, but in the BM case, the vehicle holds attitude inspite of a small gyric roll and pitch angle response to the light winds. However, the platform drifts off slowly without feedback compensation due to a steady wind component in the light winds.

Closed Loop Response to Light Winds. The closed-loop responses to light winds during hover for both cases are shown in Figure 16. Although both cases show stable response, the angular response for the BM case is clearly superior to the NBM case. Apparently, under these simulated light wind conditions, the angular response amplitudes for the NBM case appears to be small enough to be tolerated during an actual flight. The time history of the cumulative control effort (bottom right of Figure 16), shows that the case with BM actually requires less vane actuation than in the corresponding NBM case. As expected, the vane and fan actuator response did not saturate for both cases, under light winds.

Closed Loop Response to Strong Winds. The closed-loop responses to strong winds during hover for both cases are shown in Figure 17. The controller for NBM case was judiciously scaled to mitigate actuator saturation. Both cases remains stable but the angular response for the BM case is clearly superior to the NBM case. However, the significantly larger angular response amplitudes for the NBM case will likely be unacceptable or even dangerous during an actual flight. The time history of the cumulative control effort shows that the case 


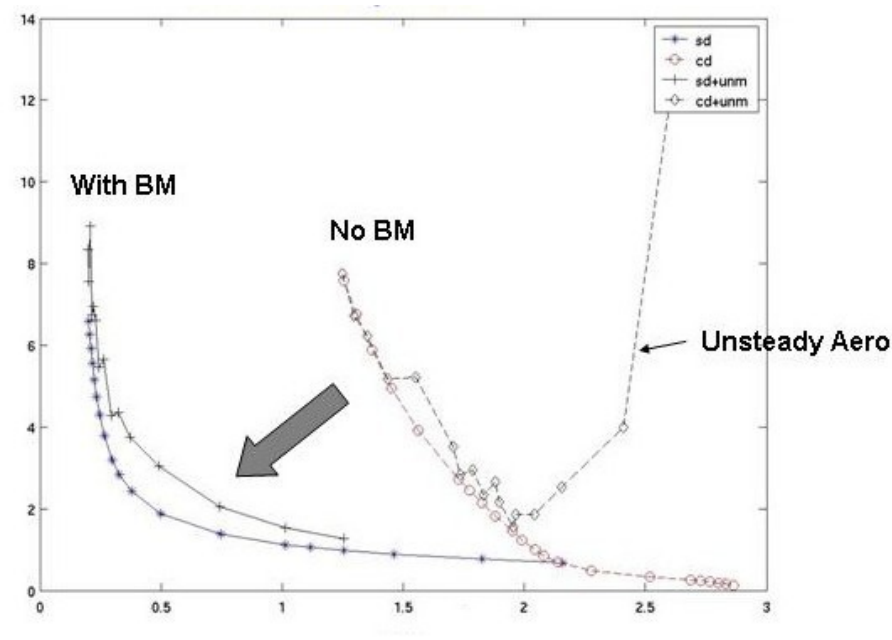

Figure 14: Performance cost (ordinate) vs actuator effort (abscissa) for an LQG set of controllers undergoing gust response during hover.

with BM actually requires less vane actuation although it gives far superior attitude hold than the corresponding NBM case. Some saturation in the vane and fan actuator resulted for the NBM case.

To examine what happens if the controller for NBM case is not judiciously scaled to mitigate actuator saturation, resulting possibly to a more agressive feedback control, an additional simulation was done with the new control law. It turns out that the close loop system loses control after a few seconds. More specifically Figure 18 shows the time histories of the vane angle and fan speed responses. The vane and fan actuators saturate resulting in loss of vehicle under strong winds.

\subsubsection{Command Tracking}

In this section, we outline a sample of simulation results of command tracking under strong winds, specifically translational velocity commands while holding a trim attitude. It should be noted that the demonstrated inherent advantage of bias momentum in endowing directional stability to the platform do not necessarily improve its maneuverability. Hence, the simulation results in this subsection is intended to examine this issue quantitatively.

For the following simulations, LQG based controllers are designed to track a trajectory path defined by a commanded translational velocities about trim with zero angular velocity. The input commands are shaped to mitigate the discontinuity in a step command. Figure 19 shows the response to translational velocity tracking commands for both cases subject to strong winds. The accompanying excursions in the angular velocities (and hence the vehicle attitude) are shown in Figure 20. It is seen that for both cases, the vehicle can successfully track translational velocity commands but $\mathrm{BM}$ case gives su-
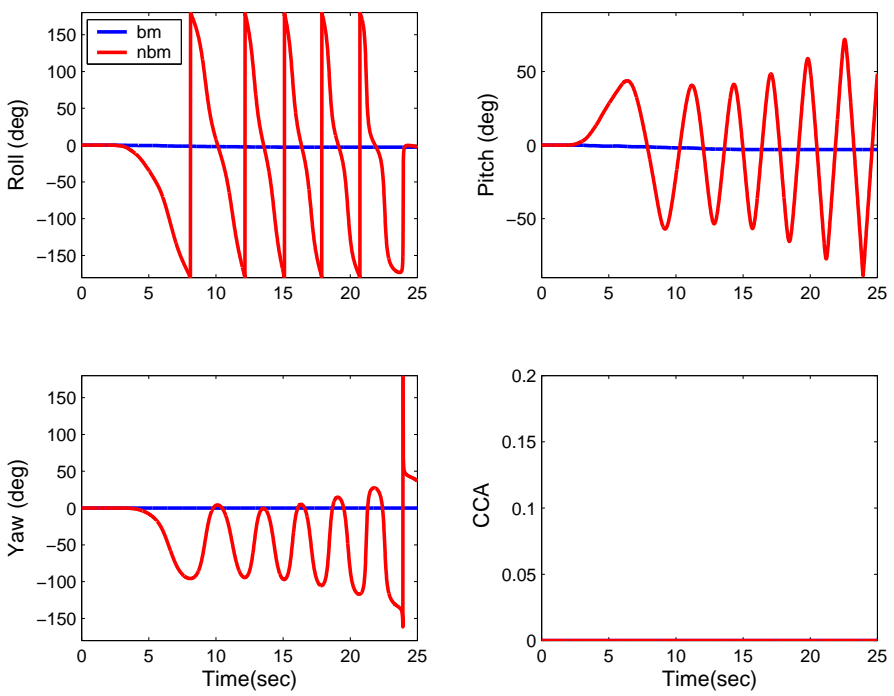

Figure 15: Open loop response to light winds, 3-2-1 Euler angles.

perior performance.

The corresponding vane and fan actuator time histories during tracking are shown in Figures 21 and 22, respectively. The case with BM clearly needs less vane and fan control activity but as observed earlier gives smaller velocity tracking errors.

\subsubsection{Robustness to payload variations}

Simulated Mass \& Inertia changes. To simulate variable payloads, we assume a step increase of 20platform. A spherical uniformly distributed mass is assumed dropped at ( $\mathrm{x}=0.15, \mathrm{y}=0, \mathrm{z}=0)$ location on the platform at 5 sec and is assumed to be dropped off from the platform at $20 \mathrm{sec}$. The inertia matrix is significantly changed as a result of the off-centered loading. This off-CG centered loading simulation is of particular interest due to its difficulty and its practical significance for a hovering platform where passengers or payloads can be loaded and unloaded with maximum flexibility without compromising vehicle stability while hovering. In this part of simulation, disturbances and measurement errors are not included to focus entirely on the effects of these payload changes.

Closed loop response during hover. Figures 23 and 24 show the position and attitude responses to mass and inertia changes for both cases. It is seen that the platform position and attitude excursions are significantly better in the BM case.

Significant differences are also noted in their corresponding vane and fan actuator histories in Figure 25. It is seen that the fan speeds and vane angles oscillates significantly and rapidly in the NBM case resulting in the platform oscillations shown previously. Unfortunately, the actual consequences on flight performance due to this 

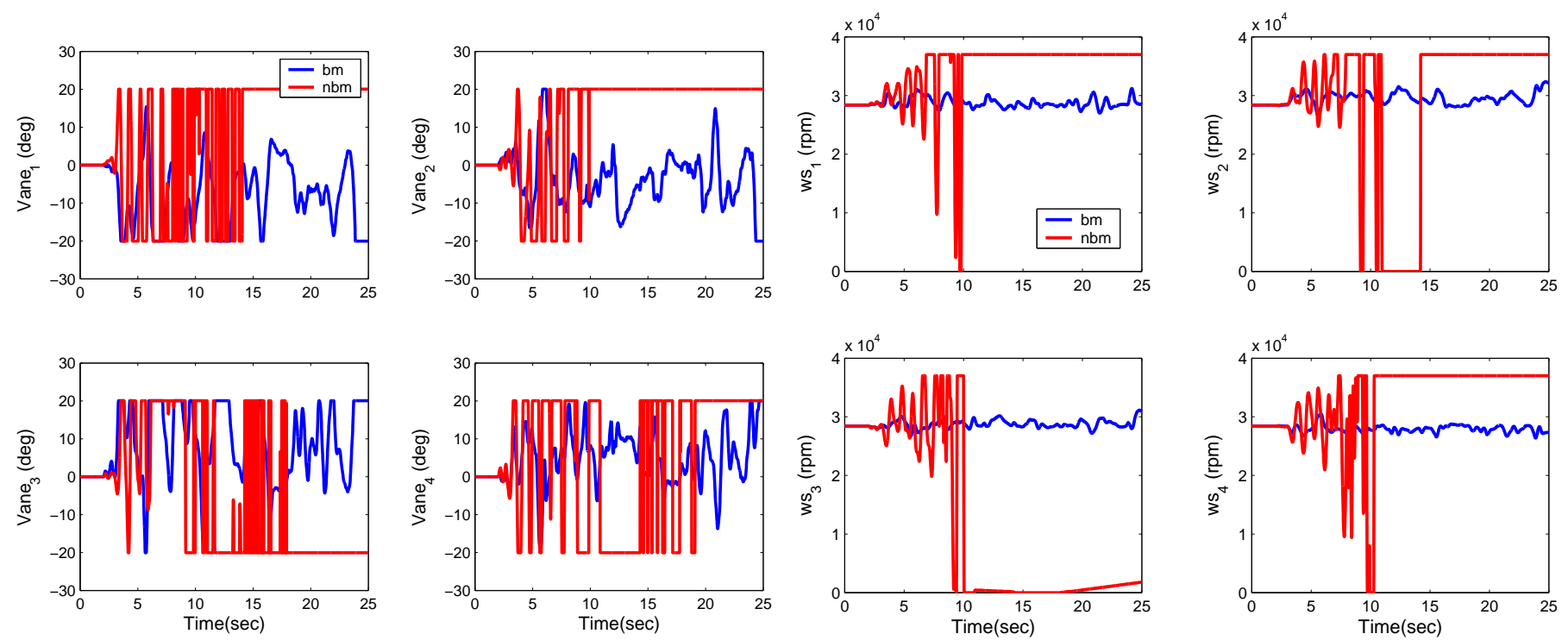

Figure 18: Closed loop response to strong winds, more agressive control.
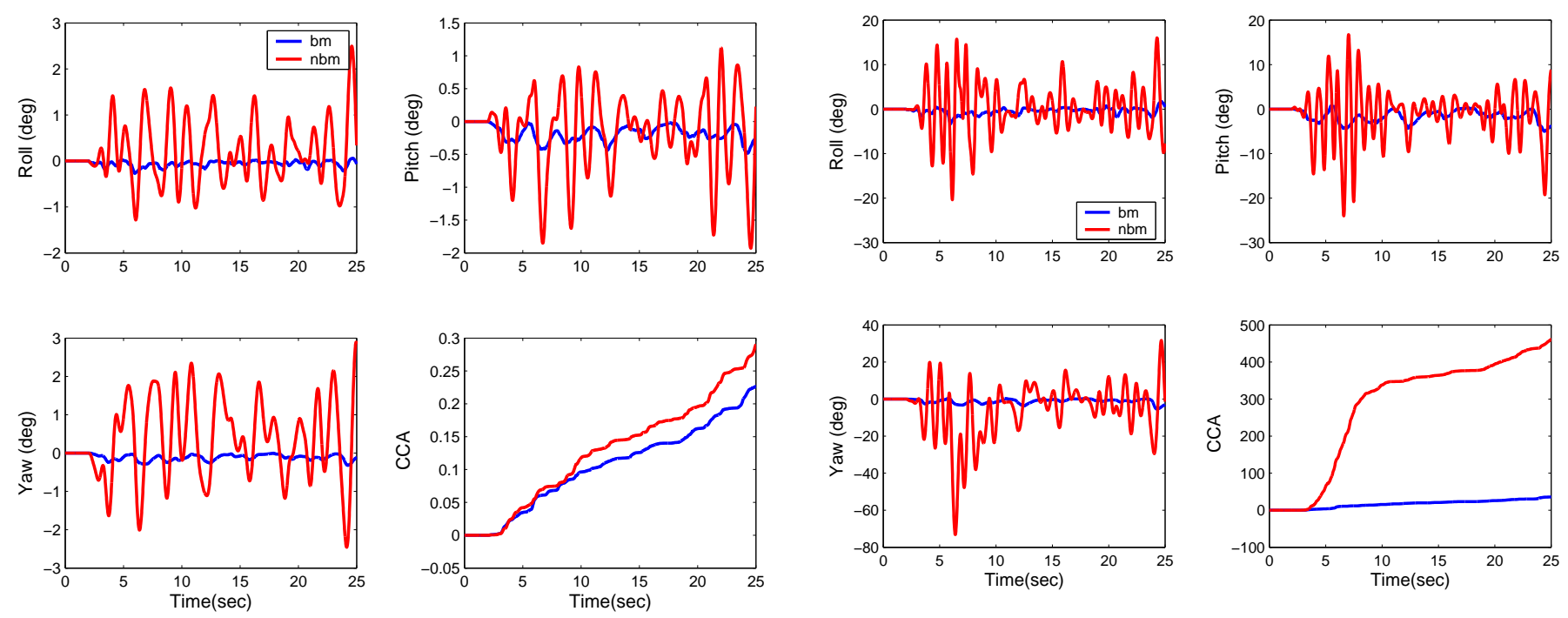

Figure 16: Closed loop response to light winds.

apparently more unsteady control vane activity for the NBM case, remains to be seen during an actual flight due to the lack of a reliable mathematical model for this class of unsteady aero.

\section{Conclusions}

A new stability and control technology, based on trying to mitigate control surface actuator induced unsteady aero effects in feedback control is proposed. Its ultimate goal is to enable statically unstable thrust levitated low-speed or hovering vehicles to operate more safely than is possible today, in turbulent conditions and under large payload variations. New results in trim, bias momentum sizing,
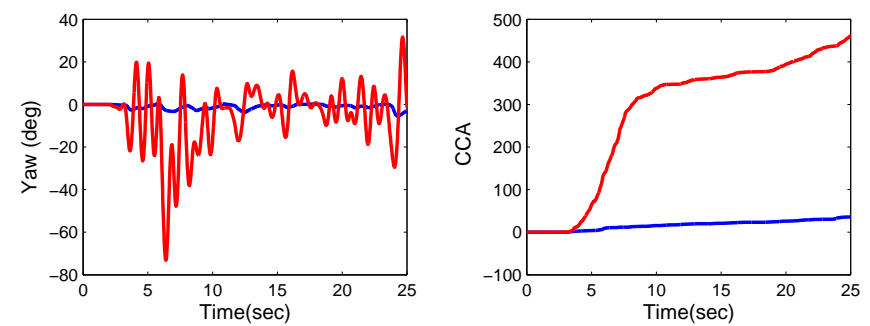

Figure 17: Closed loop response to strong winds.

and a control structure framework which integrates conventional vector thrusting with multiple wheel actuators have been developed. Simulation results indicate that generic VTOL platforms can be endowed with significant directional stability with a proper amount of bias momentum, similar to a dual-spin stabilized spacecraft but under a significantly different operating environment. The results also indicate that this open loop directional stability carries over to closed loop if the controllers are designed properly. Specifically, it was found that vehicle attitude robustness can be significantly improved (over a no-bias-momentum vehicle) during hovering, particularly under strong turbulent winds or significant payload variations. In addition, command tracking performance at low airspeeds can also be improved significantly par- 

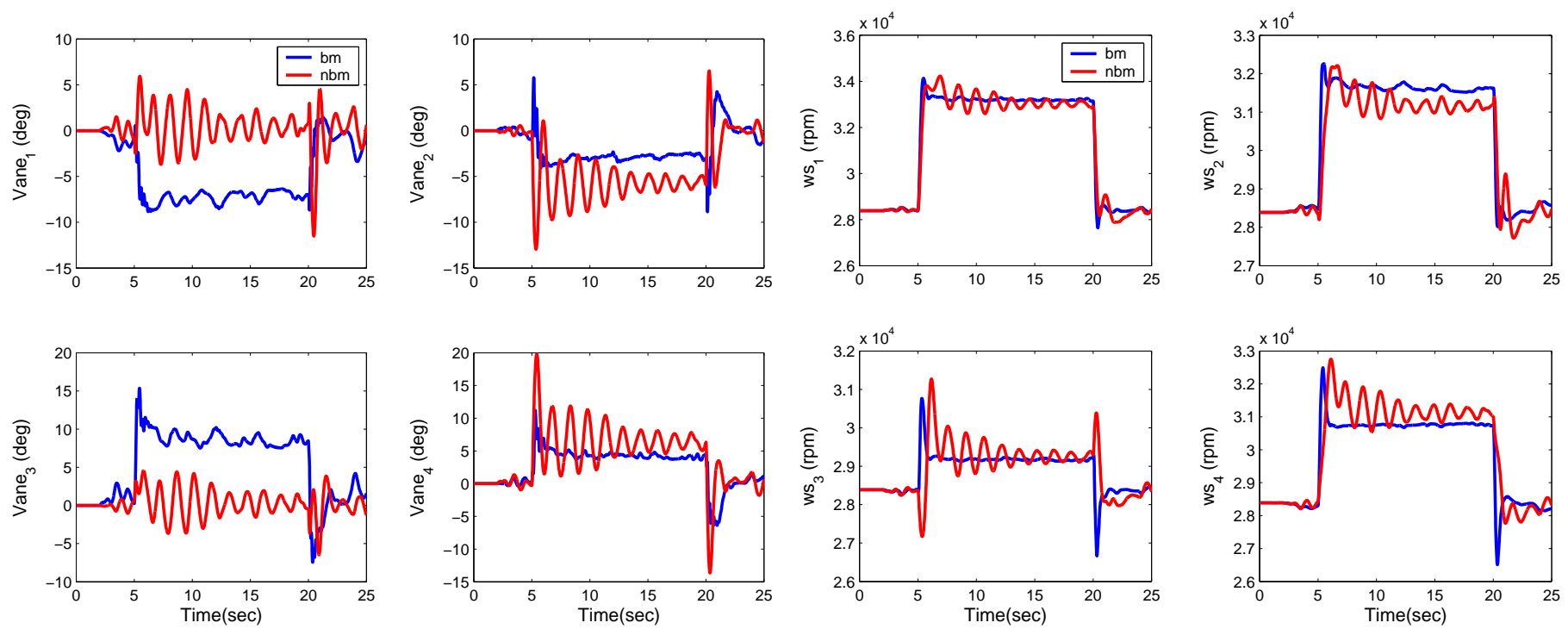

Figure 25: Actuator responses to step changes in payload.
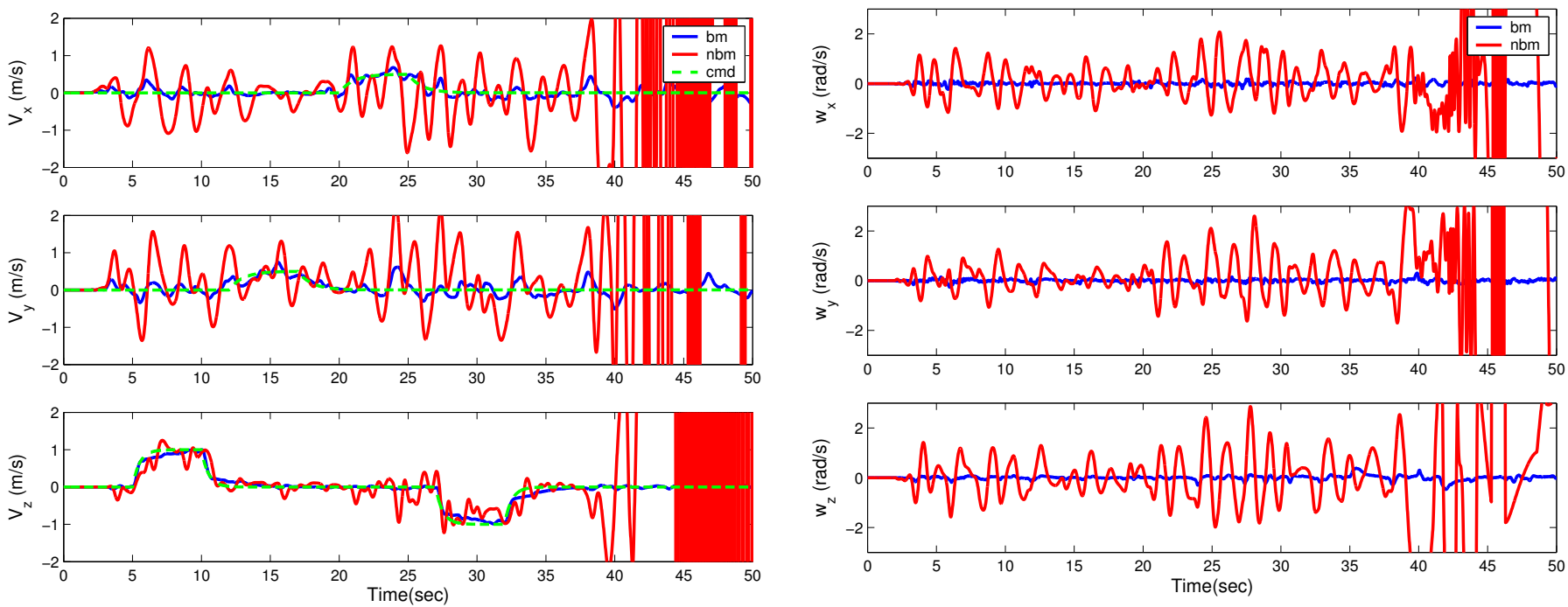

Figure 19: Vehicle velocity response during trajectory tracking.

ticularly under turbulent winds, despite the presence of somewhat complicated gyroscopic coupling. These performance improvements are due to control laws that are based on proper modeling of the system dynamics. These predicted improvements in robustness obtained from detailed simulation studies are expected to be more evident during actual flight test, with the physical presence of control induced unsteady aerodynamics.

\section{Acknowledgements}

The authors would like to thank their colleagues Elvin Ahl for developing and providing the CAD figure of ongoing NFTP test configuration, and Plesent Goode for

Figure 20: Vehicle attitude response during trajectory tracking.

giving tangible moral support for this research.

\section{References}

[1] Army Aerial \& Joint Services VTOL Rotorcraft Government/Industry/Academia Workshop, ASSP2004, Williamsburg, Virginia, 27-29 January 2004.

[2] Fleming, J., Jones, T., Gelhausen, P., and Enns, D., "Improving control system effectiveness for ducted fan VTOL UAVs operating in crosswinds", 2nd AIAA Unmanned Unlimited Systems, Technologies, and Operations - Aerospace, September 15-18, 2003, San Diego, California, AIAA Paper 2003-6514. 

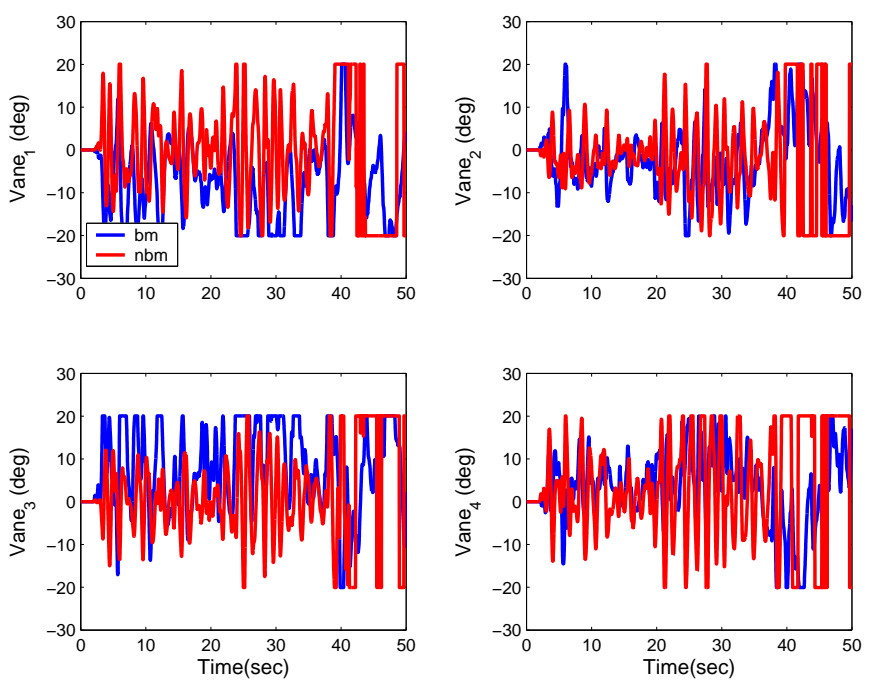

Figure 21: Vane angle response during tracking.

[3] Fleming, J., Jones, T., Lusardi, J., Gelhausen, P., and Enns, D., "Improved control of ducted fan VTOL UAVs in crosswind turbulence", AHS 4th Decennial Specialist's Conference on Aeromechanics, January 21-23, 2004, San Francisco, California.

[4] http://dmaerosafe.freeservers.com/ homepage for DM AeroPlatforms Ltd.

[5] Flow Control: Fundamentals and Practices, AIAA Professional Short Course, 22-23 June, 2002, St. Louis, Missouri.

[6] Lim,K.B., Shin,J.Y., Cooper, E.G., Moerder, D.D., Khong, T.H., and Smith,M.F., "An overview of the NASA Flying Test Platform research", AIAA Guidance, Navigation, and Control Conference and Exhibit, August 11-14, 2003, Austin, Texas. AIAA Paper 2003-5775.

[7] Hughes, P.C., Spacecraft Attitude Dynamics, John Wiley \& Sons, New York, 1986, Chapter 3.

[8] NASA Technical Paper, to appear.

[9] Spacecraft Attitude Determination and Control, Ed. J.R. Wertz, Kluwer Academic Publishing, Boston, MA, v.73, 1995 (reprint).

[10] Junkins, J.L., and Turner, J.D., Optimal Spacecraft Rotational Maneuvers, Elsevier Science Publishers, Amsterdam, Netherlands, 1986.

[11] Becker, G. and Packard, A. "Robust performance of linear parametrically varying systems using parametrically-dependent linear feedback," Systems $\& 3$ Control Letters, 23, 1994, pp. 205-215.

[12] "Theory and Application of Linear Parameter Varying Control Techniques", A. Packard, and G. J.
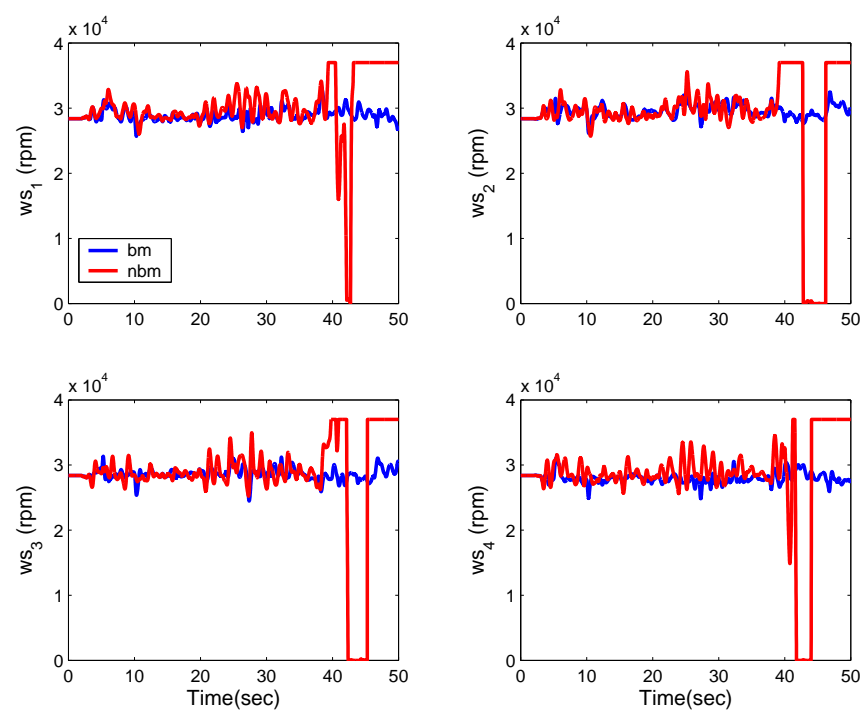

Figure 22: Fan speed response during command tracking.
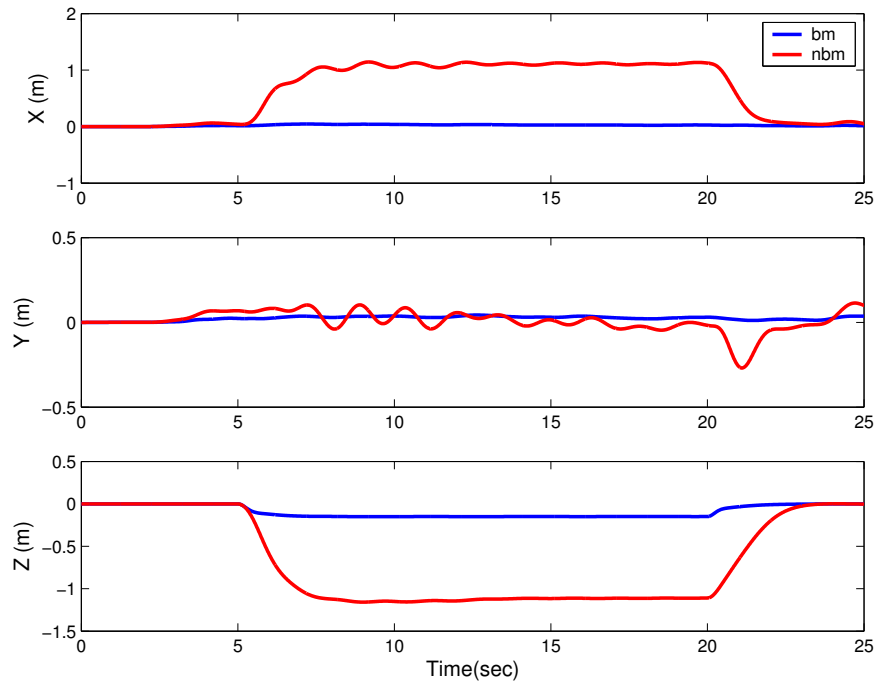

Figure 23: Position response to step changes in payload.

Balas, Tutorial Workshop at the American Control Conference, June 2-3, 1997, Albuquerque, NM.

[13] Apkarian,P., and Adams, R.J., "Advanced GainScheduling Techniques for Uncertain Systems," IEEE Transactions on Control Systems Technology, Vol. 6, No. 1, 1998, pp. 21-32.

[14] Shin, J.-Y., Balas, G.J., and Kaya, M.A., "Blending Methodology of Linear Parameter Varying Control Synthesis of F-16 Aircraft System", Journal of Guidance, Control, and Dynamics, Vol. 25, No. 6, 2002, pp. 1040-1048.

[15] Wie, B., Spacecraft Vehicle Dynamics and Control, 1998, AIAA Education Series, AIAA Inc., Reston, Virginia. 

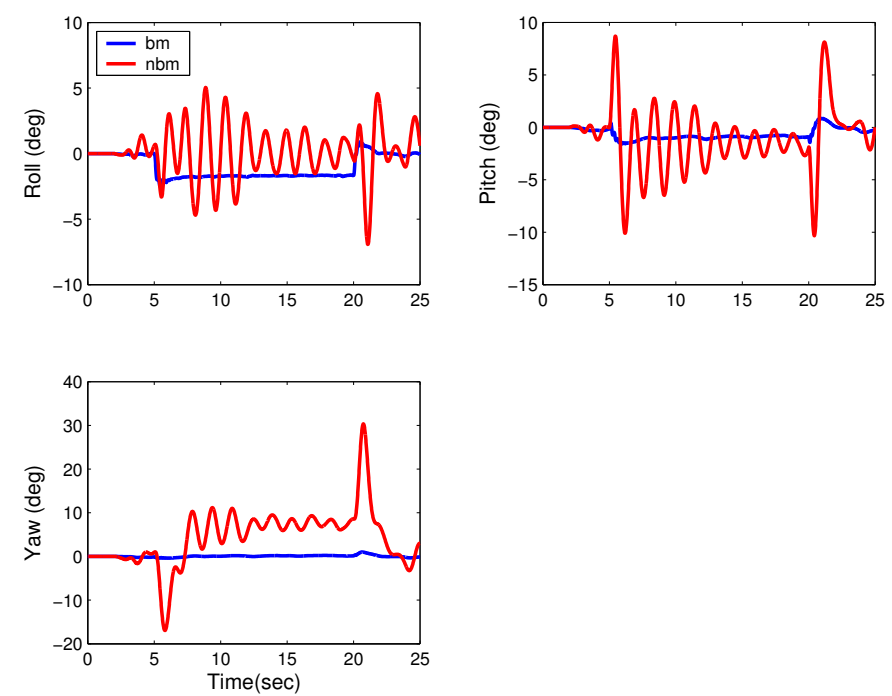

Figure 24: Attitude response to step changes in payload.

[16] Tsiotras, P., "Stabilization and Optimality Results for the Attitude Control Problem," Journal of Guidance, Control, and Dynamics, Vol. 19, No. 4, 1996, pp. $772-9$.

[17] Schaub,H., Vadali,S.R., and Junkins,J.L., "Feedback control law for variable speed control moment gyros," Journal of the Astronautical Sciences, Vol. 46, No. 3, July-Sept. 1998, pp. 307-328.

[18] George H. Saunders, Dynamics of Helicopter Flight, John Wiley \& Sons, Inc, New York, 1974.

[19] Prouty, R.W., Helicopter Aerodynamics, Vols. I-III, PJS Publications Inc, Peoria, Ill, 1985-1993. 